\title{
A FiNite ANALOG OF THE AGT RELATION I: FINITE $W$-ALGEBRAS AND QUASIMAPS' SPACES
}

\author{
ALEXANDER BRAVERMAN, BORIS FEIGIN, MICHAEL FINKELBERG AND LEONID RYBNIKOV
}

\begin{abstract}
Recently Alday, Gaiotto and Tachikawa proposed a conjecture relating 4dimensional super-symmetric gauge theory for a gauge group $G$ with certain 2-dimensional conformal field theory. This conjecture implies the existence of certain structures on the (equivariant) intersection cohomology of the Uhlenbeck partial compactification of the moduli space of framed $G$-bundles on $\mathbb{P}^{2}$. More precisely, it predicts the existence of an action of the corresponding $W$-algebra on the above cohomology, satisfying certain properties.

We propose a "finite analog" of the (above corollary of the) AGT conjecture. Namely, we replace the Uhlenbeck space with the space of based quasi-maps from $\mathbb{P}^{1}$ to any partial flag variety $G / P$ of $G$ and conjecture that its equivariant intersection cohomology carries an action of the finite $W$-algebra $U(\mathfrak{g}, e)$ associated with the principal nilpotent element in the Lie algebra of the Levi subgroup of $P$; this action is expected to satisfy some list of natural properties. This conjecture generalizes the main result of 5 when $P$ is the Borel subgroup. We prove our conjecture for $G=G L(N)$, using the works of Brundan and Kleshchev interpreting the algebra $U(\mathfrak{g}, e)$ in terms of certain shifted Yangians.
\end{abstract}

\section{INTRODUCTION}

1.1. The setup. Let $G$ be a semi-simple simply connected complex algebraic group (or, more generally, a connected reductive group whose derived group $[G, G]$ is simply connected) and let $P$ be a parabolic subgroup of $G$. We shall denote by $L$ the corresponding Levi factor. Let $B$ be a Borel subgroup of $G$ contained in $P$ and containing a maximal torus $T$ of $G$. We shall also denote by $\Lambda$ the coweight lattice of $G$ (which is the same as the lattice of cocharacters of $T)$; it has a quotient lattice $\Lambda_{G, P}=\operatorname{Hom}\left(\mathbb{C}^{*}, L /[L, L]\right)$, which can also be regarded as the lattice of characters of the center $Z(\check{L})$ of the Langlands dual group $\check{L}$. Note that $\Lambda_{G, B}=\Lambda$. The lattice $\Lambda_{G, P}$ contains canonical sub-semi-group $\Lambda_{G, P}^{+}$spanned by the images of positive coroots of $G$.

Set now $\mathcal{G}_{G, P}=G / P$. There is a natural isomorphism $H_{2}\left(\mathcal{G}_{G, P}, \mathbb{Z}\right) \simeq \Lambda_{G, P}$. Let $C$ be a smooth connected projective curve over $\mathbb{C}$. Then the degree of a map $f: C \rightarrow \mathcal{G}_{G, P}$ can be considered as an element of $\Lambda_{G, P}$; it is easy to see that actually $\operatorname{deg} f$ must lie in $\Lambda_{G, P}^{+}$. For $\theta, \theta^{\prime} \in \Lambda_{G, P}$ we shall write $\theta \geq \theta^{\prime}$ if $\theta-\theta^{\prime} \in \Lambda_{G, P}^{+}$.

Let $e_{G, P} \in \mathcal{G}_{G, P}$ denote the image of $e \in G$. Clearly $e_{G, P}$ is stable under the action of $P$ on $\mathcal{G}_{G, P}$. Let $\mathcal{M}_{G, P}$ denote the moduli space of based maps from $\left(\mathbb{P}^{1}, \infty\right)$ to $\left(\mathcal{G}_{G, P}, e_{G, P}\right)$, i.e. the moduli space of maps $\mathbb{P}^{1} \rightarrow \mathcal{G}_{G, P}$ which send $\infty$ to $e_{G, P}$. This space is acted on by the group $P \times \mathbb{C}^{*}$, where $P$ acts on $\mathcal{G}_{G, P}$ preserving the point $e_{G, P}$ and $\mathbb{C}^{*}$ acts on $\mathbb{P}^{1}$ preserving $\infty$; in particular, the reductive group $L \times \mathbb{C}^{*}$ acts on $\mathcal{M}_{G, P}$. Also for any $\theta \in \Lambda_{G, P}^{+}$let $\mathcal{M}_{G, P}^{\theta}$ denote the space of maps as above of degree $\theta$. 
For each $\theta$ as above one can also consider the space of based quasi-maps (or Zastava space in the terminology of [9], [15] and [16]; cf. also [6] for a review of quasi-maps' spaces) which we denote by $\mathcal{Q} M_{G, P}^{\theta}$. This is an affine algebraic variety containing $\mathcal{M}_{G, P}^{\theta}$ as a dense open subset. Moreover, it possesses a stratification of the form

$$
2 M_{G, P}^{\theta}=\bigcup_{0 \leq \theta^{\prime} \leq \theta} \mathcal{M}_{G, P}^{\theta^{\prime}} \times \operatorname{Sym}^{\theta-\theta^{\prime}} \mathbb{A}^{1},
$$

where for any $\gamma \in \Lambda_{G, P}^{+}$we denote by $\operatorname{Sym}^{\gamma} \mathbb{A}^{1}$ the variety of formal linear combinations $\sum \lambda_{i} x_{i}$ where $x_{i} \in \mathbb{A}^{1}$ and $\lambda_{i} \in \Lambda_{G, P}^{+}$such that $\sum \lambda_{i}=\gamma$. In most cases the variety $Q M_{G, P}^{\theta}$ is singular.

1.2. Equivariant integration. For a connected reductive group $\mathbb{G}$ with a maximal torus $\mathbb{T}$ let

$$
\mathcal{A}_{\mathbb{G}}=H_{\mathbb{G}}^{*}(p t, \mathbb{C}) .
$$

This is a graded algebra which is known to be canonically isomorphic to the algebra of $\mathbb{G}$-invariant polynomial functions on the Lie algebra $\mathbf{g}$ of $\mathbb{G}$. We shall denote by $\mathcal{K}_{\mathbb{G}}$ its field of fractions. Let now $Y$ be a variety endowed with an action of $\mathbb{G}$ such that $Y^{\mathbb{T}}$ is proper. Then as was remarked e.g. in [5] we have a well-defined integration map

$$
\int_{Y}: \mathrm{IH}_{\mathbb{G}}^{*}(Y) \rightarrow \mathcal{K}_{\mathbb{G}}
$$

which is a map of $\mathcal{A}_{\mathbb{G}}$-modules. In particular, it makes sense to consider the integral $\int_{Y} 1 \in \mathcal{K}_{\mathbb{G}}$ of the unit cohomology class.

Let us also set $\operatorname{IH}_{\mathbb{G}}^{*}(Y)_{l o c}=\operatorname{IH}_{\mathbb{G}}^{*}(Y) \otimes \mathcal{A}_{\mathbb{G}} \mathcal{K}_{\mathbb{G}}$. Then $\operatorname{IH}_{\mathbb{G}}^{*}(Y)_{l o c}$ is a finite-dimensional vector space over $\mathcal{K}_{\mathbb{G}}$ endowed with a non-degenerate $\mathcal{K}_{\mathbb{G}^{-v a l u e d}}$ (Poincaré) pairing $\langle\cdot, \cdot\rangle_{Y, \mathbb{G}}$.

In particular, all of the above is applicable to $Y=Q M_{G, P}^{\theta}$ and $\mathbb{G}=L \times \mathbb{C}^{*}$. In particular, if we let $1_{G, P}^{\theta}$ denote the unit class in the $L \times \mathbb{C}^{*}$-equivariant cohomology of $Q M_{G, P}^{\theta}$, then we can define

$$
z_{G, P}=\sum_{\theta \in \Lambda_{G, P}^{\theta}} \mathfrak{q}^{\theta} \int_{Q M_{G, P}^{\theta}} 1_{G, P}^{\theta} .
$$

This is a formal series in $\mathfrak{q} \in Z(\check{L})$ with values in the field $\mathcal{K}_{L \times \mathbb{C}^{*}}$ of $L$-invariant rational functions on $\mathfrak{l} \times \mathbb{C}$.

In fact the function $z_{G, P}$ is a familiar object in Gromov-Witten theory: it is explained in [5] that up to a simple factor $z_{G, P}$ is the so called equivariant $J$-function of $\mathcal{G}_{G, P}$ (cf. Section 6 of [5]). In particular, this function was studied from many different points of view ( cf. [19], 20], [5] for the case when $P$ is the Borel subgroup). It was conjectured in [5], [6] that the function $z_{G, P}$ should have an interpretation in terms of representation theory related to the Langlands dual Lie algebra $\check{\mathfrak{g}}$. More generally, let us set

$$
\begin{array}{r}
\mathrm{IH}_{G, P}^{\theta}=\mathrm{IH}_{L \times \mathbb{C}^{*}}\left(2 M_{G, P}^{\theta}\right)_{l o c}, \quad \mathrm{IH}_{G, P}=\bigoplus_{\theta \in \Lambda_{G, P}^{+}} \mathrm{IH}_{G, P}^{\theta}, \\
\langle\cdot, \cdot\rangle_{G, P}^{\theta}=\langle\cdot, \cdot\rangle_{Q M_{G, P}^{\theta}, L \times \mathbb{C}^{*}}, \quad\langle\cdot, \cdot\rangle_{G, P}=\bigoplus_{\theta \in \Lambda_{G, P}^{+}}(-1)^{\langle\theta, \check{\rho}\rangle}\langle\cdot, \cdot\rangle_{G, P}^{\theta},
\end{array}
$$


where $\check{\rho}$ denotes the half-sum of the positive roots of $\mathfrak{g}$, and we view $\theta$ as the positive integral linear combination of (the images of ) the simple coroots out of $\mathfrak{l}$.

Then one would like to interpret the $\Lambda_{G, P}^{+}$-graded vector space $\mathrm{IH}_{G, P}$ together with the intersection pairing $\langle\cdot, \cdot\rangle$ and the unit cohomology vectors $1_{G, P}^{\theta} \in \mathrm{IH}_{G, P}^{\theta}$ in such terms. A complete answer to this problem in the case when $P$ is a Borel subgroup was given in [5]. The purpose of this paper is to suggest a conjectural answer in the general case and to prove this conjecture for $G=G L(N)$. The relevant representation theory turns out to be the representation theory of finite $W$-algebras which we recall in Section 2, We should also note that our conjecture is motivated by the so called Alday-Gaiotto-Tachikawa (or AGT) conjecture [2] which relates 4-dimensional super-symmetric gauge theory to certain 2-dimensional conformal field theory (more precisely, one may view some part of the AGT conjecture as an affine version of our conjecture; this point of view is explained in Section 66). In fact, the current work grew out of an attempt to create an approach to the AGT conjecture in terms of geometric representation theory. We hope to pursue this point of view in future publications.

1.3. The main conjecture. In the remainder of this introduction we are going to give a more precise formulation of our main conjecture and indicate the idea of the proof for $G=G L(N)$. To do this, let us first recall the corresponding result from [5] dealing with the case when $P$ is a Borel subgroup. In what follows we shall denote it by $B$ instead of $P$.

First, it is shown in [5] that the Lie algebra $\check{\mathfrak{g}}$ acts naturally on $\mathrm{IH}_{G, B}$. Moreover, this action has the following properties. First of all, let us denote by $\langle\cdot, \cdot\rangle_{G, B}$ the direct sum of the pairings $(-1)^{\langle\theta, \check{\rho}\rangle}\langle\cdot, \cdot\rangle_{G, B}^{\theta}$.

Recall that the Lie algebra $\check{\mathfrak{g}}$ has its triangular decomposition $\check{\mathfrak{g}}=\check{\mathfrak{n}}_{+} \oplus \check{\mathfrak{h}} \oplus \check{\mathfrak{n}}_{-}$. Let $\kappa: \check{\mathfrak{g}} \rightarrow \check{\mathfrak{g}}$ denote the Cartan anti-involution which interchanges $\check{\mathfrak{n}}_{+}$and $\check{\mathfrak{n}}_{-}$and acts as identity on $\check{\mathfrak{h}}$. For each $\lambda \in \mathfrak{h}=(\check{\mathfrak{h}})^{*}$ we denote by $M(\lambda)$ the corresponding Verma module with highest weight $\lambda$; this is a module generated by a vector $v_{\lambda}$ with (the only) relations

$$
t\left(v_{\lambda}\right)=\lambda(t) v_{\lambda} \quad \text { for } t \in \check{\mathfrak{h}} \text { and } \quad n\left(v_{\lambda}\right)=0 \quad \text { for } n \in \check{\mathfrak{n}}_{+} .
$$

Theorem 1.4. (1) $\mathrm{IH}_{G, B}$ (with the above action) becomes isomorphic to $M(\lambda)$, where $\lambda=-\frac{a}{\hbar}-\rho$.

(2) $\mathrm{IH}_{G, B}^{\theta} \subset \mathrm{IH}_{G, B}$ is the $-\frac{a}{\hbar}-\rho-\theta$-weight space of $\mathrm{IH}_{G, B}$.

(3) For each $g \in \check{\mathfrak{g}}$ and $v, w \in \mathrm{IH}_{G, B}$ we have

$$
\langle g(v), w\rangle_{G, B}=\langle v, \kappa(g) w\rangle_{G, B} .
$$

(4) The vector $\sum_{\theta} 1_{G, B}^{\theta}$ (lying is some completion of $\mathrm{IH}_{G, B}$ ) is a Whittaker vector (i.e. a $\mathfrak{n}_{+}$-eigen-vector) for the above action.

As a corollary we get that the function $\mathfrak{q}^{\frac{a}{\hbar}} z_{G, B}$ is an eigen-function of the quantum Toda hamiltonians associated with $\mathfrak{\mathfrak { g }}$ with eigen-values determined (in the natural way) by $a$ (we refer the reader to [13 for the definition of (affine) Toda integrable system and its relation with Whittaker functions). In fact, in [5] a similar statement is proved also when $G$ is replaced by the corresponding affine Kac-Moody group — cf. Section 6 for more detail.

Our main conjecture gives a generalization of the statements 1)-3) above to arbitrary $P$. Namely, to any nilpotent element $e \in \mathfrak{g}$ one can associate the so called finite $W$-algebra 
$U(\check{\mathfrak{g}}, e)$. We recall the definition in Section 2.1 (this definition is such that when $e=0$ we have $U(\check{\mathfrak{g}}, e)=U \check{\mathfrak{g}}$ and when $e$ is regular, then $U(\check{\mathfrak{g}}, e)$ is the center of $U \check{\mathfrak{g}})$. Roughly speaking, we conjecture that analogs of 1)-3) hold when $U \check{\mathfrak{g}}$ is replaced by $U\left(\check{\mathfrak{g}}, e_{\check{L}}\right)$ (we refer the reader to Section 2 for the definition of Verma module and Whittaker vectors for finite $W$-algebras). The main purpose of this paper is to formulate this conjecture more precisely and to prove it for $G$ of type $A$.

1.5. Organization of the paper. The paper is organized as follows: in Section 2 we recall basic definitions about finite $W$-algebras for general $G$; we also recall the basic results about parabolic quasi-maps' spaces and formulate our main conjecture. In Section 3 we recall the results of Brundan and Kleshchev who interpret finite $W$-algebras in type $A$ using certain shifted Yangians and in Section 5 we discuss the notion of Whittaker vectors for finite $W$-algebras from this point of view. In Section 4 we use it in order to prove our main conjecture for $G=S L(N)$ (and any parabolic). One important ingredient in the proof is this: we replace the intersection cohomology of parabolic quasi-maps' spaces by the ordinary cohomology of a small resolution of those spaces (which we call parabolic Laumon spaces). Finally in Section 6 we discuss the relation between the above results and the AGT conjecture.

1.6. Acknowledgements. We are grateful to A. Molev for the explanation of the results of [18. We are also grateful to J. Brundan, A. Kleschev and I. Losev for their explanations about $W$-algebras. Thanks are due to A. Tsymbaliuk for the careful reading of the first draft of this note and spotting several mistakes, and to S. Gukov, D. Maulik, A. Nietzke, A. Okounkov, V. Pestun, Y. Tachikawa for very helpful discussions on the subject. A. B. was partially supported by the NSF grants DMS-0854760 and DMS-0901274. B. F, M. F., and L. R. were partially supported by the RFBR grant 09-01-00242, the Ministry of Education and Science of Russian Federation grant No. 2010-1.3.1-111-017-029, and the AG Laboratory HSE, RF government grant, ag. 11.G34.31.0023, and HSE science foundation grant 10-01-0078.

\section{Finite $W$-ALGEBRAS}

2.1. $W$-algebra. Let $e$ be a principal nilpotent element of the Levi subalgebra $\mathfrak{l} \subset \mathfrak{g}$. Let $U(\mathfrak{g}, e)$ denote the finite $W$-algebra associated to $e$, see e.g. [11. We recall its definition for the readers' convenience. Choose an $\mathfrak{s l}_{2}$-triple $(e, h, f)$ in $\mathfrak{g}$. We introduce a grading on $\mathfrak{g}$ by eigenvalues of $\operatorname{ad}_{h}$ :

$$
\mathfrak{g}=\bigoplus_{i \in \mathbb{Z}} \mathfrak{g}(i), \mathfrak{g}(i):=\{\xi \in \mathfrak{g}:[h, \xi]=i \xi\} .
$$

The Killing form $(\cdot, \cdot)$ on $\mathfrak{g}$ allows to identify $\mathfrak{g}$ with the dual space $\mathfrak{g}^{*}$. Let $\chi=(e, ?)$ be an element of $\mathfrak{g}^{*}$ corresponding to $e$. Note that $\chi$ defines a symplectic form $\omega_{\chi}$ on $\mathfrak{g}(-1)$ as follows: $\omega_{\chi}(\xi, \eta):=\langle\chi,[\xi, \eta]\rangle$. We fix a Lagrangian subspace $l \subset \mathfrak{g}(-1)$ with respect to $\omega_{\chi}$, and set $\mathfrak{m}:=l \oplus \bigoplus_{i \leq-2} \mathfrak{g}(i)$. We define the affine subspace $\mathfrak{m}_{\chi} \subset U \mathfrak{g}$ as follows: $\mathfrak{m}_{\chi}:=\{\xi-\langle\chi, \xi\rangle, \xi \in \mathfrak{m}\}$. Finally, we define the $W$-algebra $U(\mathfrak{g}, e):=\left(U \mathfrak{g} / U \mathfrak{g} \cdot \mathfrak{m}_{\chi}\right)^{\text {ad } \mathfrak{m}}:=$ $\left\{a+U \mathfrak{g} \cdot \mathfrak{m}_{\chi}:[\mathfrak{m}, a] \subset U \mathfrak{g} \cdot \mathfrak{m}_{\chi}\right\}$. It is easy to see that

$$
U(\mathfrak{g}, e)=\text { End }_{U \mathfrak{g}}\left(U \mathfrak{g} \underset{U \mathfrak{m}}{\otimes} \mathbb{C}_{\chi}\right),
$$


where $\mathbb{C}_{\chi}$ denotes the natural 1-dimensional module over $\mathfrak{m}$ corresponding to the character $\chi$. In this description the algebra structure on $U(\mathfrak{g}, e)$ becomes manifest.

It is equipped with the Kazhdan filtration $\mathrm{F}_{0} U(\mathfrak{g}, e) \subset \mathrm{F}_{1} U(\mathfrak{g}, e) \subset \ldots$, see e.g. section 3.2 of 11. We recall its definition for the readers' convenience. We denote the standard PBW filtration on $U \mathfrak{g}$ (by the order of a monomial) by $\mathrm{F}_{i}^{s t} U \mathfrak{g}$. The Kazhdan filtration on $U \mathfrak{g}$ is defined by $\mathrm{F}_{i} U \mathfrak{g}:=\sum_{2 k+j \leq i} \mathrm{~F}_{k}^{s t} U \mathfrak{g} \cap U \mathfrak{g}(j)$ where $U \mathfrak{g}(j)$ is the eigenspace of $\operatorname{ad}_{h}$ on $U \mathfrak{g}$ with eigenvalue $j$. Being a subquotient of $U \mathfrak{g}$, the $W$-algebra $U(\mathfrak{g}, e)$ inherits the Kazhdan filtration $\mathrm{F}_{i} U(\mathfrak{g}, e)$.

We also consider the shifted Kazhdan filtration $F_{i} U(\mathfrak{g}, e):=\mathrm{F}_{i+1} U(\mathfrak{g}, e)$, and we define the $\mathbb{C}[\hbar]$-algebra $U^{\hbar}(\mathfrak{g}, e)$ as the Rees algebra of the filtered algebra $\left(U(\mathfrak{g}, e), F_{\bullet}\right)$. Abusing notation, we will sometimes call $U^{\hbar}(\mathfrak{g}, e)$ just a $W$-algebra.

Let us extend the scalars to the field $\mathcal{K}:=\mathcal{K}_{\check{L} \times \mathbb{C}^{*}}$, and let $\hbar \in \mathcal{K}$ stand for the generator of $H_{\mathbb{C}^{*}}^{2}(p t, \mathbb{Z})$. Thus $\mathcal{K}=\mathbb{C}\left(\mathfrak{t}^{*} / W_{L} \times \mathbb{A}^{1}\right)$ where $W_{L}$ stands for the Weyl group of $\mathfrak{l}$, and $\mathbb{A}^{1}$ is the affine line with coordinate $\hbar$.

Given a point $\Lambda \in \mathfrak{t}^{*} / W_{L}$ we consider the Verma module $M\left(-\hbar^{-1} \Lambda, e\right)$ over $U^{\hbar}(\mathfrak{g}, e) \otimes$ $\mathcal{K} \simeq U(\mathfrak{g}, e) \otimes \mathcal{K}$ introduced in sections 4.2 and 5.1 of [11]. As $\Lambda \in \mathfrak{t}^{*} / W_{L}$ varies, these modules form a family over $\mathbb{C}\left[\mathfrak{t}^{*} / W_{L} \times\left(\mathbb{A}^{1}-0\right)\right]$. Localizing to $\mathcal{K}$ we obtain the universal Verma module $M(\mathfrak{g}, e)$ over $U(\mathfrak{g}, e) \otimes \mathcal{K}$. Note that a certain $\rho$-shift is incorporated into the definition of $M(\mathfrak{g}, e)$, cf. the text right after Lemma 5.1 of [1] 1 .

In what follows we shall often abbreviate $M:=M(\mathfrak{g}, e)$.

2.2. Whittaker vector. Let $\mathfrak{t}^{e}$ stand for the centralizer of $e$ in $\mathfrak{t}$. Recall from [11] that the collection of nonzero weights of $\mathfrak{t}^{e}$ on $\mathfrak{g}$ is called a restricted root system $\Phi^{e}$, and the weights on $\mathfrak{p}^{\prime}:=\bigoplus_{i \geq 0} \mathfrak{g}(i)$ form a positive root system $\Phi_{+}^{e} \subset \Phi^{e}$. Let $I^{e}$ be the set of simple roots, i.e. positive roots which are not positive linear combinations of other positive roots. According to Theorem 6 of [10], the simple roots form a base of $\left(\mathfrak{t}^{e}\right)^{\vee}$.

Let us choose a linear embedding $\Theta: \mathfrak{g}^{e} \hookrightarrow U^{\hbar}(\mathfrak{g}, e)$ as in Theorem 3.6 of [11]. For a simple root $\alpha \in I^{e}$, we consider the corresponding weight space $\Theta\left(\mathfrak{g}_{\alpha}^{e}\right)$. The Kazhdan filtration induces the increasing filtration on the root space $\Theta\left(\mathfrak{g}_{\alpha}^{e}\right)$. We define a positive integer $m_{\alpha}$ so that $\mathrm{F}_{m_{\alpha}} \Theta\left(\mathfrak{g}_{\alpha}^{e}\right)=\Theta\left(\mathfrak{g}_{\alpha}^{e}\right)$, but $\mathrm{F}_{m_{\alpha}-1} \Theta\left(\mathfrak{g}_{\alpha}^{e}\right) \neq \Theta\left(\mathfrak{g}_{\alpha}^{e}\right)$. The following conjecture holds for $\mathfrak{g}$ of type $A$ by the work of J. Brundan and A. Kleshchev (cf. Section 3.7 below), and for all exceptional types according to computer calculations by J. Brundan (private communication):

Conjecture 2.3. $\operatorname{dim} \mathrm{F}_{m_{\alpha}} \Theta\left(\mathfrak{g}_{\alpha}^{e}\right) / \mathrm{F}_{m_{\alpha}-1} \Theta\left(\mathfrak{g}_{\alpha}^{e}\right)=1$.

Definition 2.4. A linear functional $\psi$ on $\bigoplus_{\alpha \in I^{e}} \Theta\left(\mathfrak{g}_{\alpha}^{e}\right)$ is called regular if for any $\alpha \in I^{e}$ we have $\psi\left(\mathrm{F}_{m_{\alpha}-1} \Theta\left(\mathfrak{g}_{\alpha}^{e}\right)\right)=0$ but $\psi\left(\mathrm{F}_{m_{\alpha}} \Theta\left(\mathfrak{g}_{\alpha}^{e}\right)\right) \neq 0$.

If Conjecture 2.3 is true, then $T^{e}$ acts simply transitively on the set of regular functionals.

Definition 2.5. Given a regular functional $\psi$ on $\bigoplus_{\alpha \in I^{e}} \Theta\left(\mathfrak{g}_{\alpha}^{e}\right)$, a $\psi$-eigenvector $\mathfrak{w}$ in a completion $\prod_{\theta \in \Lambda_{\breve{G}, \check{P}}} M_{\theta}$ of the universal Verma module $M$ is called a $\psi$-Whittaker vector.

\footnotetext{
${ }^{1}$ The definition of the module $M(\mathfrak{g}, e)$ from [11] is (unfortunately) quite involved and we are not going to recall it here. On the other hand for $\mathfrak{g}=\mathfrak{g l}(N)$ there is another (in some sense, more explicit) definition of this module which we are going to recall in Section 3 .
} 
2.6. Shapovalov form. Let $\sigma$ stand for the Cartan antiinvolution of $\mathfrak{g}$ identical on $\mathfrak{t}$. Let $w_{0}^{\mathfrak{l}}$ stand for the adjoint action of a representative of the longest element of the Weyl group of the Levi subalgebra $\mathfrak{l}$. Then the composition $w_{0}^{\mathfrak{l}} \sigma$ preserves $e$ and everything else entering the definition of the finite $W$-algebra and gives rise to an antiisomorphism $U^{\hbar}(\mathfrak{g}, e) \stackrel{\sim}{\longrightarrow} \bar{U}^{\hbar}(\mathfrak{g}, e)$ where $\bar{U}^{\hbar}(\mathfrak{g}, e)$ (see Section 2.2 of [11]) is defined just as $U^{\hbar}(\mathfrak{g}, e)$, only with left ideals replaced by right ideals. Composing this antiisomorphism with the isomorphism $\bar{U}^{\hbar}(\mathfrak{g}, e) \stackrel{\sim}{\longrightarrow} U^{\hbar}(\mathfrak{g}, e)$ of Corollary 2.9 of [11] we obtain an antiinvolution $\varsigma$ of $U^{\hbar}(\mathfrak{g}, e)$.

Definition 2.7. The Shapovalov bilinear form $(\cdot, \cdot)$ on the universal Verma module $M$ with values in $\mathcal{K}$ is the unique bilinear form such that $(x, y u)=(\varsigma(y) x, u)$ for any $x, u \in M, y \in$ $U^{\hbar}(\mathfrak{g}, e)$ with value 1 on the highest vector.

2.8. Parabolic Zastava spaces and finite $W$-algebras: the main conjecture. We are now ready to formulate our main conjectures. We are going to change slightly the notations. Namely, the symbols $G, P, L, \mathfrak{g}, \mathfrak{p}, \mathfrak{l}$ will denote the same things as in the introduction. However, we are now going to denote by $e$ the principal nilpotent element in the Langlands dual Lie algebra $\check{\mathfrak{l}} \subset \mathfrak{g}$; similarly, $M$ will now denote the universal Verma module over $U(\check{\mathfrak{g}}, e)$. Note that in this case $M$ becomes naturally graded by $\Lambda_{G, P}^{+}$. Also, the universal coefficient field $\mathcal{K}$ is now nothing else but the field $\mathcal{K}_{L \times \mathbb{C}^{*}}$ which appeared in the introduction. With these conventions we formulate the following

Conjecture 2.9. (1) There is an isomorphism $\Psi$ of $\Lambda_{G, P}$-graded $\mathcal{K}$-vector spaces $\mathrm{IH}_{G, P}$ and $M$; in particular, there is a natural $U^{\hbar}(\check{\mathfrak{g}}, e)$-action on $\mathrm{IH}_{G, P}$.

(2) The isomorphism $\Psi$ takes the vector $\sum_{\theta \in \Lambda_{G, P}} 1^{\theta} \in \prod_{\theta \in \Lambda_{G, P}} \mathrm{IH}_{G, P}^{\theta}$ to a $\psi$-Whittaker vector $\mathfrak{w} \in \prod_{\theta \in \Lambda_{G, P}} M_{\theta}$ for certain regular functional $\psi$.

(3) For $x, u \in V_{\theta}$ we have $(\Psi(x), \Psi(u))=(-1)^{|\theta|}\langle x, u\rangle_{G, P}^{\theta}$.

In what follows we shall give a more precise formulation of this conjecture when $\mathfrak{g}$ is of type $A$ (in that case we shall also prove the conjecture). In particular, we shall specify the regular functional $\psi$ in that case.

\section{Shifted Yangians and Finite $W$-ALgebras}

In this Section we recall an explicit realization of finite $W$-algebras in type $A$ using shifted Yangians (due to Brundan and Kleshchev).

3.1. Shifted Yangian. Let $\pi=\left(p_{1}, \ldots, p_{n}\right)$ where $p_{1} \leq p_{2} \leq \ldots \leq p_{n}$, and $p_{1}+\ldots+p_{n}=$ $N$. Recall the shifted Yangian $Y_{\pi}^{\hbar}\left(\mathfrak{g l}_{n}\right)$ introduced by J. Brundan and A. Kleshchev (see [12] and [18]). It is an associative $\mathbb{C}[\hbar]$-algebra with generators $\mathrm{d}_{i}^{(r)}, i=1, \ldots, n, r \geq 1 ; \mathrm{f}_{i}^{(r)}, i=$ $1, \ldots, n-1, r \geq 1 ; \mathrm{e}_{i}^{(r)}, i=1, \ldots, n-1, r \geq p_{i+1}-p_{i}+1$, subject to the following relations

$$
\begin{gathered}
{\left[\mathbf{d}_{i}^{(r)}, \mathbf{d}_{j}^{(s)}\right]=0} \\
{\left[\mathrm{e}_{i}^{(r)}, \mathrm{f}_{j}^{(s)}\right]=-\delta_{i j} \hbar \sum_{t=0}^{r+s-1} \mathrm{~d}_{i}^{\prime(t)} \mathrm{d}_{i+1}^{(r+s-t-1)}}
\end{gathered}
$$


where $\mathrm{d}_{i}^{(0)}:=1$, and the elements $\mathrm{d}_{i}^{\prime(r)}$ are found from the relations $\sum_{t=0}^{r} \mathrm{~d}_{i}^{(t)} \mathrm{d}_{i}^{\prime(r-t)}=$ $\delta_{r 0}, r=0,1, \ldots$;

$$
\begin{gathered}
{\left[\mathrm{d}_{i}^{(r)}, \mathrm{e}_{j}^{(s)}\right]=\hbar\left(\delta_{i j}-\delta_{i, j+1}\right) \sum_{t=0}^{r-1} \mathrm{~d}_{i}^{(t)} \mathrm{e}_{j}^{(r+s-t-1)},} \\
{\left[\mathrm{d}_{i}^{(r)}, \mathrm{f}_{j}^{(s)}\right]=\hbar\left(\delta_{i, j+1}-\delta_{i j}\right) \sum_{t=0}^{r-1} \mathrm{f}_{j}^{(r+s-t-1)} \mathrm{d}_{i}^{(t)},} \\
{\left[\mathrm{e}_{i}^{(r)}, \mathrm{e}_{i}^{(s+1)}\right]-\left[\mathrm{e}_{i}^{(r+1)}, \mathrm{e}_{i}^{(s)}\right]=\hbar\left(\mathrm{e}_{i}^{(r)} \mathrm{e}_{i}^{(s)}+\mathrm{e}_{i}^{(s)} \mathrm{e}_{i}^{(r)}\right),} \\
{\left[\mathrm{f}_{i}^{(r+1)}, \mathrm{f}_{i}^{(s)}\right]-\left[\mathrm{f}_{i}^{(r)}, \mathrm{f}_{i}^{(s+1)}\right]=\hbar\left(\mathrm{f}_{i}^{(r)} \mathrm{f}_{i}^{(s)}+\mathrm{f}_{i}^{(s)} \mathrm{f}_{i}^{(r)}\right),} \\
{\left[\mathrm{e}_{i}^{(r)}, \mathrm{e}_{i+1}^{(s+1)}\right]-\left[\mathrm{e}_{i}^{(r+1)}, \mathrm{e}_{i+1}^{(s)}\right]=-\hbar \mathrm{e}_{i}^{(r)} \mathrm{e}_{i+1}^{(s)},} \\
{\left[\mathrm{f}_{i}^{(r+1)}, \mathrm{f}_{i+1}^{(s)}\right]-\left[\mathrm{f}_{i}^{(r)}, \mathrm{f}_{i+1}^{(s+1)}\right]=-\hbar \mathrm{f}_{i+1}^{(s)} \mathrm{f}_{i}^{(r)},} \\
{\left[\mathrm{e}_{i}^{(r)}, \mathrm{e}_{j}^{(s)}\right]=0 \quad \text { if }|i-j|>1,} \\
{\left[\mathrm{f}_{i}^{(r)}, \mathrm{f}_{j}^{(s)}\right]=0 \quad \text { if }|i-j|>1,} \\
\left.\left[\mathrm{e}_{i}^{(r)},\left[\mathrm{e}_{i}^{(s)}, \mathrm{e}_{j}^{(t)}\right]\right]+\left[\mathrm{f}_{i}^{(s)}, \mathrm{f}_{j}^{(t)}\right]\right]+\left[\mathrm{f}_{i}^{(s)},\left[\mathrm{f}_{i}^{(r)}, \mathrm{f}_{j}^{(t)}\right]\right]=0 \\
\text { if }|i-j|=1
\end{gathered}
$$

We introduce the generating series

$$
\begin{gathered}
\mathrm{d}_{k}(u)=1+\sum_{s=1}^{\infty} \mathrm{d}_{k}^{(s)} \hbar^{-s+1} u^{-s}, \quad \mathrm{e}_{k}(u)=\sum_{s=p_{k+1}-p_{k}+1}^{\infty} \mathrm{e}_{k}^{(s)} \hbar^{-s+1} u^{-s}, \\
\mathrm{f}_{k}(u):=\sum_{s=1}^{\infty} \mathrm{f}_{k}^{(s)} \hbar^{-s+1} u^{-s} .
\end{gathered}
$$

Finally, we define $\mathrm{a}_{k}(u):=\mathrm{d}_{1}(u) \mathrm{d}_{2}(u-1) \ldots \mathrm{d}_{k}(u-k+1), \mathrm{A}_{k}(u):=u^{p_{1}}(u-1)^{p_{2}} \ldots(u-$ $k+1)^{p_{k}} \mathrm{a}_{k}(u)$, and also

$$
\begin{aligned}
& \mathrm{B}_{k}(u):=(u-k+1)^{p_{k+1}-p_{k}} \mathrm{~A}_{k}(u) \mathrm{e}_{k}(u-k+1), \\
& \mathrm{C}_{k}(u):=\mathrm{f}_{k}(u-k+1) \mathrm{A}_{k}(u) .
\end{aligned}
$$


3.2. $W$-algebra $U^{\hbar}(\mathfrak{g}, e)$ and its universal Verma module. Let $\mathfrak{p} \subset \mathfrak{g}:=\mathfrak{g l}_{N}=$ End $(W)$ be the parabolic subalgebra preserving the flag $0 \subset W_{1} \subset \ldots \subset W_{n-1} \subset W$. Here $W=\left\langle w_{1}, \ldots, w_{N}\right\rangle$, and $W_{i}=\left\langle w_{1}, \ldots, w_{p_{1}+\ldots+p_{i}}\right\rangle$. Let $e$ be a principal nilpotent element of a Levi factor $\mathfrak{l}$ of $\mathfrak{p}$. Let $U(\mathfrak{g}, e)$ denote the finite $W$-algebra associated to $e$, see e.g. [12]. It is equipped with the Kazhdan filtration $\mathrm{F}_{0} U(\mathfrak{g}, e) \subset \mathrm{F}_{1} U(\mathfrak{g}, e) \subset \ldots$, see e.g. section 3.2 of [12. We also consider the shifted Kazhdan filtration $F_{i} U(\mathfrak{g}, e):=\mathrm{F}_{i+1} U(\mathfrak{g}, e)$, and we define the $\mathbb{C}[\hbar]$-algebra $U^{\hbar}(\mathfrak{g}, e)$ as the Rees algebra of the filtered algebra $\left(U(\mathfrak{g}, e), F_{\bullet}\right)$. Abusing notation, we will sometimes call $U^{\hbar}(\mathfrak{g}, e)$ just a $W$-algebra.

According to section 3.4 of [12], $U^{\hbar}(\mathfrak{g}, e) \otimes_{\mathbb{C}[\hbar]} \mathbb{C}(\hbar)$ is the quotient of $Y_{\pi}^{\hbar}\left(\mathfrak{g l}_{n}\right) \otimes_{\mathbb{C}[\hbar]} \mathbb{C}(\hbar)$ by the relations $\mathrm{d}_{1}^{(r)}=0, r>p_{1}$.

Let us denote the standard coordinates in the diagonal Cartan subalgebra $\mathfrak{t}$ of $\mathfrak{g l}_{N}$ by $x_{1}, \ldots, x_{N}$. Then the universal Verma module $M=M\left(\mathfrak{g l}_{N}, e\right)$ over $U^{\hbar}(\mathfrak{g}, e) \otimes \mathcal{K}$ is a vector space over the field $\mathcal{K}$ of rational functions in $\hbar, x_{1}, \ldots, x_{N}$ symmetric in the groups $\left(x_{1}, \ldots, x_{p_{1}}\right),\left(x_{p_{1}+1}, \ldots, x_{p_{1}+p_{2}}\right), \ldots,\left(x_{p_{1}+\ldots+p_{n-1}+1}, \ldots, x_{N}\right)$. Let us consider the field extension $\mathcal{K} \subset \mathcal{K}^{\prime}:=\mathbb{C}\left(\mathfrak{t}^{*} \times \mathbb{A}^{1}\right)=\mathbb{C}\left(\hbar, x_{1}, \ldots, x_{N}\right)$. We denote by $M^{\prime}=M^{\prime}\left(\mathfrak{g l}_{N}, e\right):=$ $M \otimes_{\mathcal{K}} \mathcal{K}^{\prime}$ the universal Verma module with the extended scalars.

3.3. The Gelfand-Tsetlin module. According to [18, the module $M^{\prime}$ admits a rather explicit description. More precisely, the authors of [18] construct a Gelfand-Tsetlin module $V$ over $U^{\hbar}(\mathfrak{g}, e) \otimes \mathcal{K}^{\prime}$ equipped with a Gelfand-Tsetlin base numbered by the Gelfand-Tsetlin patterns, and write down explicitly the matrix coefficients of the generators of $U^{\hbar}(\mathfrak{g}, e)$ in this base. We recall these results here. To a collection $\underline{\vec{d}}=\left(d_{i j}^{(a)}\right), n-1 \geq i \geq j, p_{j} \geq a \geq 1$, we associate a Gelfand-Tsetlin pattern $\Lambda=\Lambda(\underline{\vec{d}}):=\left(\lambda_{i j}^{(a)}\right), n \geq i \geq j, p_{j} \geq a \geq 1$, as follows: $\lambda_{n j}^{(a)}:=\hbar^{-1} x_{p_{1}+\ldots+p_{j-1}+a}+j-1, n \geq j \geq 1 ; \lambda_{i j}^{(a)}:=-\hbar^{-1} \mathbf{p}_{i j}^{(a)}+j-1, n-1 \geq i \geq j \geq 1$ (see (3.15)). The corresponding base element $\xi_{\Lambda}=\xi_{\Lambda(\underline{\vec{d}})}$ will be denoted by $\xi_{\vec{d}}$ for short. Thus, the set $\left\{\xi_{\vec{d}}\right\}$ (over all collections $\underline{\vec{d}}$ ) forms a basis of $V$.

We have used the following notation: given $\underline{\vec{d}}$,

$$
\mathrm{p}_{i k}^{(l)}:=\hbar d_{i k}^{(l)}-x_{p_{1}+\ldots+p_{k-1}+l}, 1 \leq l \leq p_{i}
$$

Also, for $n \geq i \geq j$ we introduce the monic polynomials $\lambda_{i j}(u):=\left(u+\lambda_{i j}^{(1)}\right) \ldots\left(u+\lambda_{i j}^{\left(p_{j}\right)}\right)$.

Finally, we define the action of the generators of $U^{\hbar}\left(\mathfrak{g l}_{N}, e\right)$ on $V$ by their matrix elements in the Gelfand-Tsetlin base:

$$
\mathrm{e}_{i\left[\vec{d}, \underline{d}^{\prime}\right]}^{(s)}=-\hbar^{-1-p_{i}}\left(\mathrm{p}_{i j}^{(a)}-i \hbar\right)^{s-1-p_{i+1}+p_{i}} \prod_{\substack{k \leq i, b \leq p_{k} \\(k, b) \neq(j, a)}}\left(\mathrm{p}_{i j}^{(a)}-\mathrm{p}_{i k}^{(b)}\right)^{-1} \prod_{k \leq i+1} \prod_{b \leq p_{k}}\left(\mathrm{p}_{i j}^{(a)}-\mathrm{p}_{i+1, k}^{(b)}\right),
$$

if $d_{i j}^{(a) \prime}=d_{i j}^{(a)}-1$ for certain $j \leq i$;

$$
\mathrm{f}_{i\left[\overrightarrow{\underline{d}}, \vec{d}^{\prime}\right]}^{(s)}=\hbar^{-1+p_{i}}\left(\mathbf{p}_{i j}^{(a)}+(1-i) \hbar\right)^{s-1} \prod_{\substack{k \leq i, b \leq p_{k} \\(k, b) \neq(j, a)}}\left(\mathrm{p}_{i j}^{(a)}-\mathrm{p}_{i k}^{(b)}\right)^{-1} \prod_{k \leq i-1} \prod_{b \leq p_{k}}\left(\mathrm{p}_{i j}^{(a)}-\mathrm{p}_{i-1, k}^{(b)}\right),
$$

if $d_{i j}^{(a) \prime}=d_{i j}^{(a)}+1$ for certain $j \leq i$. All the other matrix coefficients of $\mathrm{e}_{i}^{(s)}, \mathrm{f}_{i}^{(s)}$ vanish.

The following Proposition is taken from [18]. 
Proposition 3.4. The formulas (3.16), (3.17) give rise to the action of $U^{\hbar}\left(\mathfrak{g l}_{N}, e\right) \otimes \mathcal{K}^{\prime}$ on $V$. Moreover, $\mathrm{A}_{i}(u), \mathrm{C}_{i}(u), \mathrm{B}_{i}(u)$ are polynomials in $u$ of degrees $p_{1}+\ldots+p_{i}$ (resp. $\left.p_{1}+\ldots+p_{i}-1, p_{1}+\ldots+p_{i}-1\right)$ such that

$$
\mathrm{A}_{i}(u) \xi_{\underline{\vec{d}}}=\lambda_{i 1}(u) \ldots \lambda_{i i}(u-i+1) \xi_{\overrightarrow{\vec{d}}}
$$

for $i=1, \ldots, n$, and

$$
\begin{aligned}
& \mathrm{B}_{i}\left(\hbar^{-1} \mathbf{p}_{i j}^{(a)}\right) \xi_{\underline{\vec{d}}}=-\lambda_{i+1,1}\left(\hbar^{-1} \mathbf{p}_{i j}^{(a)}\right) \lambda_{i+1,2}\left(\hbar^{-1} \mathbf{p}_{i j}^{(a)}-1\right) \ldots \lambda_{i+1, i+1}\left(\hbar^{-1} \mathbf{p}_{i j}^{(a)}-i\right) \xi_{\underline{\vec{d}}+\delta_{i j}^{(a)}}, \\
& \mathrm{C}_{i}\left(\hbar^{-1} \mathrm{p}_{i j}^{(a)}\right) \xi_{\vec{d}}=\lambda_{i-1,1}\left(\hbar^{-1} \mathbf{p}_{i j}^{(a)}\right) \lambda_{i-1,2}\left(\hbar^{-1} \mathrm{p}_{i j}^{(a)}-1\right) \ldots \lambda_{i-1, i-1}\left(\hbar^{-1} \mathrm{p}_{i j}^{(a)}-i+2\right) \xi_{\vec{d}-\delta_{i j}^{(a)}},
\end{aligned}
$$

for $i=1, \ldots, n-1$, where $\underline{\vec{d}} \pm \delta_{i j}^{(a)}$ is obtained from $\underline{\vec{d}}$ by replacing $d_{i j}^{(a)}$ by $d_{i j}^{(a)} \pm 1$.

Proof. The formulas (3.19) and (3.16) (resp. (3.20) and (3.17)) are equivalent by the Lagrange interpolation. So if suffices to prove that the formulas (3.18), (3.19), (3.20) give rise to the action of $U^{\hbar}\left(\mathfrak{g l}_{N}, e\right) \otimes \mathcal{K}^{\prime}$ on $V$. Now $V$ admits a certain integral form over $\mathbb{C}\left[\hbar^{ \pm 1}, x_{1}, \ldots, x_{N}\right]$ which can be specialized to the values of parameters $x_{i}$ satisfying certain integrality and positivity conditions. These specializations admit finite-dimensional subspaces spanned by certain finite subsets of the Gelfand-Tsetlin base. Theorem 4.1 of [18] describes the action of $U^{\hbar}\left(\mathfrak{g l}_{N}, e\right)$ in these finite-dimensional subspaces by the formulas (3.18), (3.19), (3.20). It follows that the action of generators given by (3.18), (3.19), (3.20) in these subspaces satisfies the relations of $U^{\hbar}\left(\mathfrak{g l}_{N}, e\right)$. For each given $\underline{\vec{d}}$, both $\underline{\vec{d}}$ and $\underline{\vec{d}} \pm \delta_{i j}^{(a)}$ enter the above finite subsets for quite a few specializations: more precisely, the set of special values of $x_{1}, \ldots, x_{N}$ such that both $\underline{\vec{d}}$ and $\underline{\vec{d}} \pm \delta_{i j}^{(a)}$ enter the corresponding finite subsets is Zariski dense in $\mathfrak{t}^{*} \times \mathbb{A}^{1}$. It follows that the relations of $U^{\hbar}\left(\mathfrak{g l}_{N}, e\right)$ are satisfied for all values of $x_{1}, \ldots, x_{N}$.

Proposition 3.5. The Gelfand-Tsetlin module $V$ is isomorphic to the universal Verma module with extended scalars $M^{\prime}$.

Proof. First, $V$ is an irreducible module over $U^{\hbar}\left(\mathfrak{g l}_{N}, e\right) \otimes \mathcal{K}^{\prime}$. In effect, by Proposition 3.4 the Gelfand-Tsetlin subalgebra of $U^{\hbar}\left(\mathfrak{g l}_{N}, e\right) \otimes \mathcal{K}^{\prime}$ generated by $\mathrm{d}_{i}^{(r)}$ acts diagonally in the Gelfand-Tsetlin base with pairwise distinct eigenvalues. Therefore, it suffices to check the following two things:

(1) for each $\underline{\vec{d}}$ there are indices $i, s$ such that $\mathrm{f}_{i}^{(s)} \xi_{\vec{d}} \neq 0$;

(2) for each $\underline{\vec{d}} \neq 0$ there are indices $i, s$ such that $\underline{\mathrm{e}}_{i}^{(s)} \xi_{\overrightarrow{\vec{d}}} \neq 0$.

Both follow directly from the formulas (3.16), (3.17).

Second, $M^{\prime}$ is an irreducible module over $U^{\hbar}\left(\mathfrak{g l}_{N}, e\right) \otimes \mathcal{K}^{\prime}$. In effect, for a general highest weight $\Lambda \in \mathfrak{t}^{*} / W_{L}$ the Verma module $M\left(-\hbar^{-1} \Lambda, e\right)$ is irreducible according to [11]. Hence the universal Verma module is irreducible as well.

Now to construct the desired isomorphism $M^{\prime} \stackrel{\sim}{\longrightarrow} V$ it suffices to produce a nonzero homomorphism $M^{\prime} \rightarrow V$. By the universal property of Verma modules, it suffices to identify the highest weights of $M^{\prime}$ and $V$. By the argument in the proof of Theorem 5.5 of [11], $\mathrm{d}_{i}^{(r)}$ acts on the highest vector of $M^{\prime}$ by multiplication by the $r$ th elementary symmetric 
polynomial in the variables $i-1-\hbar^{-1} x_{j}, p_{1}+\ldots+p_{i-1}+1 \leq j \leq p_{1}+\ldots+p_{i}$. On the other hand, it follows from the formula (3.18) that $\mathrm{d}_{i}(u)$ acts on the highest vector $\xi_{0}$ by multiplication by $u^{-p_{i}} \prod_{j=p_{1}+\ldots+p_{i-1}+1}^{p_{1}+\ldots+p_{i}}\left(u+i-1-\hbar^{-1} x_{j}\right)$. The coincidence of highest weights completes the proof of the proposition.

Recall that the Galois group of $\mathcal{K}^{\prime}$ over $\mathcal{K}$ is $W_{L}$. By the irreducibility of $U^{\hbar}\left(\mathfrak{g l}_{N}, e\right) \otimes$ $\mathcal{K}^{\prime}$-module $V$, there is a unique semilinear action of $W_{L}$ on $V$ intertwining the action of $U^{\hbar}\left(\mathfrak{g l}_{N}, e\right) \otimes \mathcal{K}^{\prime}$, and trivial on the highest vector $\xi_{0}$.

Corollary 3.6. The universal Verma module $M\left(\mathfrak{g l}_{N}, e\right)$ is isomorphic to $V^{W_{L}}$.

3.7. The characters of positive subalgebra. Recall the notations of [11. So $\mathfrak{t}$ stands for the diagonal Cartan subalgebra of $\mathfrak{g}$, and $\mathfrak{t}^{e}$ stands for the centralizer of $e$ in $\mathfrak{t}$, and $\mathfrak{g}^{e}$ stands for the centralizer of $e$ in $\mathfrak{g}$. The collection $\Phi^{e}$ of non-zero weights of $\mathfrak{t}^{e}$ on $\mathfrak{g}$ is a restricted root system, see e.g. section 3.1 of [11]. The roots appearing in $\mathfrak{p}^{\prime} \subset \mathfrak{g}$ form a system $\Phi_{+}^{e} \subset \Phi^{e}$ of positive roots. Let us denote by $\mathfrak{g}_{+}^{e} \subset \mathfrak{g}^{e}$ the subspace spanned by the positive root vectors. Recall a linear space embedding $\Theta: \mathfrak{g}^{e} \hookrightarrow U^{\hbar}(\mathfrak{g}, e)$ of Theorem 3.6 of [11. We define $U_{+}^{\hbar}(\mathfrak{g}, e)$ as the subalgebra of $U^{\hbar}(\mathfrak{g}, e)$ generated by $\Theta\left(\mathfrak{g}_{+}^{e}\right)$. In terms of the shifted Yangian, $U_{+}^{\hbar}(\mathfrak{g}, e)$ is generated by $\left\{\mathrm{e}_{i}^{(s)}\right\}, 1 \leq i \leq n-1, s \geq p_{i+1}-p_{i}+1$.

We are interested in the (additive) characters of $U_{+}^{\hbar}(\mathfrak{g}, e)$, that is maximal ideals of $U_{+}^{\hbar}(\mathfrak{g}, e)^{\text {ad }}:=U_{+}^{\hbar}(\mathfrak{g}, e) /\left[U_{+}^{\hbar}(\mathfrak{g}, e), U_{+}^{\hbar}(\mathfrak{g}, e)\right]$. We have $U_{+}^{\hbar}(\mathfrak{g}, e)^{\text {ad }} \simeq \operatorname{Sym}\left(\Theta\left(\mathfrak{g}_{+}^{e}\right)^{\text {ad }}\right)$ where $\Theta\left(\mathfrak{g}_{+}^{e}\right)^{\text {ad }}:=\Theta\left(\mathfrak{g}_{+}^{e}\right) / \Theta\left(\mathfrak{g}_{+}^{e}\right) \cap\left[U_{+}^{\hbar}(\mathfrak{g}, e), U_{+}^{\hbar}(\mathfrak{g}, e)\right]$. In terms of roots, $\Theta\left(\mathfrak{g}_{+}^{e}\right)^{\text {ad }}$ is spanned by the simple positive root spaces in $\Theta\left(\mathfrak{g}_{+}^{e}\right)$. In terms of the shifted Yangian, $\Theta\left(\mathfrak{g}_{+}^{e}\right)^{\text {ad }}$ is spanned by $\left\{\mathrm{e}_{i}^{(s)}\right\}, 1 \leq i \leq n-1, p_{i+1}-p_{i}+1 \leq s \leq p_{i+1}$.

The Kazhdan filtration induces the increasing filtration on the root space $\Theta\left(\mathfrak{g}_{\alpha}^{e}\right)$ for a simple positive root $\alpha \in \Phi_{+}^{e}$. In terms of the shifted Yangian, $\mathrm{F}_{r} \Theta\left(\mathfrak{g}_{\alpha_{i}}^{e}\right)$ is spanned by $\left\{\mathrm{e}_{i}^{(s)}\right\}, 1 \leq i \leq n-1, r \geq s \geq p_{i+1}-p_{i}+1$. For a simple root $\alpha$ we define $m_{\alpha}$ so that $\mathrm{F}_{m_{\alpha}} \Theta\left(\mathfrak{g}_{\alpha}^{e}\right)=\Theta\left(\mathfrak{g}_{\alpha}^{e}\right)$, but $\mathrm{F}_{m_{\alpha}-1} \Theta\left(\mathfrak{g}_{\alpha}^{e}\right) \neq \Theta\left(\mathfrak{g}_{\alpha}^{e}\right)$. Clearly, for $\alpha=\alpha_{i}$ we have $m_{\alpha_{i}}=p_{i+1}$.

We say that an additive character $\chi: U_{+}^{\hbar}(\mathfrak{g}, e) \rightarrow \mathbb{C}(\hbar)$, that is a linear function $\Theta\left(\mathfrak{g}_{+}^{e}\right)^{\text {ad }} \rightarrow \mathbb{C}(\hbar)$, is regular if $\chi\left(\mathrm{F}_{m_{\alpha}-1} \Theta\left(\mathfrak{g}_{\alpha}^{e}\right)\right)=0$, but $\chi\left(\mathrm{F}_{m_{\alpha}} \Theta\left(\mathfrak{g}_{\alpha}^{e}\right)\right) \neq 0$ for any simple root $\alpha$. Let $T^{e}$ stand for the centralizer of $e$ in the diagonal torus of $G$. Then the adjoint action of $T^{e}$ on the set of regular characters is transitive. We specify one particular regular character in terms of the shifted Yangian: $\chi_{\hbar}\left(\mathrm{e}_{i}^{(s)}\right)=0$ for $p_{i+1}-p_{i}+1 \leq s<p_{i+1}$, and $\chi_{\hbar}\left(\mathrm{e}_{i}^{\left(p_{i+1}\right)}\right)=\hbar^{-1}$.

Definition 3.8. The Whittaker vector $\mathfrak{w} \in \widehat{M}$ in a completion of the universal Verma module (the product of the weight spaces) is the unique eigenvector for $U_{+}^{\hbar}(\mathfrak{g}, e)$ with the eigenvalue $\chi_{\hbar}$ whose highest weight component coincides with the highest vector. For a weight $\underline{d}$ we denote by $\mathfrak{w}_{\underline{d}}$ the weight $\underline{d}$ component of $\mathfrak{w}$.

3.9. The Shapovalov form in terms of shifted Yangians. We consider the antiinvolution $\varsigma: Y_{\pi}^{\hbar}\left(\mathfrak{g l}_{n}\right) \rightarrow Y_{\pi}^{\hbar}\left(\mathfrak{g l}_{n}\right)$ taking $\mathrm{d}_{i}^{(s)}$ to $\mathrm{d}_{i}^{(s)}$, and $\mathrm{f}_{i}^{(s)}$ to $\mathrm{e}_{i}^{\left(s+p_{i+1}-p_{i}\right)}$. This is nothing else than the composition of isomorphism (2.35) of [12] and anti-isomorphism (2.39) of [12]. It descends to the same named antiinvolution $\varsigma: U^{\hbar}(\mathfrak{g}, e) \rightarrow U^{\hbar}(\mathfrak{g}, e)$. According to section 3.5 of [12], this antiinvolution can be alternatively described as follows. Let $\sigma$ stand 
for the Cartan antiinvolution of $\mathfrak{g}$ (transposition). Let $w_{0}^{\mathfrak{l}}$ stand for the adjoint action of a representative of the longest element of the Weyl group of the Levi subalgebra $\mathfrak{l}$. Then the composition $w_{0}^{\mathfrak{l}} \sigma$ preserves $e$ and everything else entering the definition of the finite $W$ algebra and gives rise to an antiisomorphism $U^{\hbar}(\mathfrak{g}, e) \stackrel{\sim}{\longrightarrow} \bar{U}^{\hbar}(\mathfrak{g}, e)$ where $\bar{U}^{\hbar}(\mathfrak{g}, e)$ (see Section 2.2 of [11]) is defined just as $U^{\hbar}(\mathfrak{g}, e)$, only with left ideals replaced by right ideals. Composing this antiisomorphism with the isomorphism $\bar{U}^{\hbar}(\mathfrak{g}, e) \stackrel{\sim}{\longrightarrow} U^{\hbar}(\mathfrak{g}, e)$ of Corollary 2.9 of [11] we obtain an antiinvolution of $U^{\hbar}(\mathfrak{g}, e)$. This antiinvolution coincides with $\varsigma$.

Definition 3.10. The Shapovalov bilinear form $(\cdot, \cdot)$ on the universal Verma module $M$ with values in $\mathbb{C}\left(\hbar, x_{1}, \ldots, x_{n}\right)$ is the unique bilinear form such that $(x, y u)=(\varsigma(y) x, u)$ for any $x, u \in M, y \in U^{\hbar}(\mathfrak{g}, e)$, with value 1 on the highest vector.

\section{Parabolic Laumon spaces and Correspondences: Proof of the main CONJECTURE FOR $G=G L(n)$}

In this section we prove Conjecture 2.9 for $G=G L_{N}$. Note that in this case $G \simeq \check{G}, P \simeq$ $\check{P}, L \simeq \check{L}, T \simeq \check{T}$.

4.1. We recall the setup of [17]. Let $\mathbf{C}$ be a smooth projective curve of genus zero. We fix a coordinate $z$ on $\mathbf{C}$, and consider the action of $\mathbb{C}^{*}$ on $\mathbf{C}$ such that $v(z)=v^{-1} z$. We have $\mathbf{C}^{\mathbb{C}^{*}}=\{0, \infty\}$.

We consider an $N$-dimensional vector space $W$ with a basis $w_{1}, \ldots, w_{N}$. This defines a Cartan torus $T \subset G=G L_{N}=\operatorname{Aut}(W)$ acting on $W$ as follows: for $T \ni \underline{t}=\left(t_{1}, \ldots, t_{N}\right)$ we have $\underline{t}\left(w_{i}\right)=t_{i} w_{i}$.

4.2. We fix an $n$-tuple of positive integers $p_{1} \leq p_{2} \leq \ldots \leq p_{n}$ such that $p_{1}+\ldots+p_{n}=N$. Let $P \subset G$ be a parabolic subgroup preserving the flag $0 \subset W_{1}:=\left\langle w_{1}, \ldots, w_{p_{1}}\right\rangle \subset W_{2}:=$ $\left\langle w_{1}, \ldots, w_{p_{1}+p_{2}}\right\rangle \subset \ldots \subset W_{n-1}:=\left\langle w_{1}, \ldots w_{p_{1}+\ldots+p_{n-1}}\right\rangle \subset W_{n}:=W$. Let $G / P$ be the corresponding partial flag variety.

Given an $(n-1)$-tuple of nonnegative integers $\underline{d}=\left(d_{1}, \ldots, d_{n-1}\right)$, we consider the Laumon's parabolic quasiflags' space $Q_{\underline{d}}$, see [21, 4.2. It is the moduli space of flags of locally free subsheaves

$$
0 \subset \mathcal{W}_{1} \subset \ldots \subset \mathcal{W}_{n-1} \subset \mathcal{W}=W \otimes \mathcal{O}_{\mathbf{C}}
$$

such that $\operatorname{rank}\left(\mathcal{W}_{k}\right)=p_{1}+\ldots+p_{k}$, and $\operatorname{deg}\left(\mathcal{W}_{k}\right)=-d_{k}$.

It is known to be a smooth connected projective variety of dimension $\operatorname{dim}(G / P)+$ $\sum_{i=1}^{n-1} d_{i}\left(p_{i}+p_{i+1}\right)$, see [21], 2.10.

4.3. We consider the following locally closed subvariety $\mathfrak{Q}_{\underline{d}} \subset \mathcal{Q}_{\underline{d}}$ (parabolic quasiflags based at $\infty \in \mathbf{C}$ ) formed by the flags

$$
0 \subset \mathcal{W}_{1} \subset \ldots \subset \mathcal{W}_{n-1} \subset \mathcal{W}=W \otimes \mathcal{O}_{\mathbf{C}}
$$

such that $\mathcal{W}_{i} \subset \mathcal{W}$ is a vector subbundle in a neighbourhood of $\infty \in \mathbf{C}$, and the fiber of $\mathcal{W}_{i}$ at $\infty$ equals the span $\left\langle w_{1}, \ldots, w_{p_{1}+\ldots+p_{i}}\right\rangle \subset W$.

It is known to be a smooth connected quasiprojective variety of dimension $\sum_{i=1}^{n-1} d_{i}\left(p_{i}+\right.$ $\left.p_{i+1}\right)$. Moreover, there is a natural proper morphism $\mathfrak{Q}_{\underline{d}} \rightarrow \mathcal{Q} M_{\bar{G}, P}^{d}$ and according to A. Kuznetsov, this morphism is a small resolution of singularities (cf. Remark after Theorem 7.3 of $[9])$, so that $H_{T \times \mathbb{C}^{*}}^{\bullet}\left(\mathfrak{Q}_{\underline{d}}\right)=\operatorname{IH}_{T \times \mathbb{C}^{*}}^{\bullet}\left(Q M_{G, P}^{\frac{d}{G}}\right)$, and $H_{L \times \mathbb{C}^{*}}^{\bullet}\left(\mathfrak{Q}_{\underline{d}}\right)=\operatorname{IH}_{L \times \mathbb{C}^{*}}^{\bullet}\left(Q M_{G, P}\right)$. 
4.4. Fixed points. The group $G \times \mathbb{C}^{*}$ acts naturally on $Q_{\underline{d}}$, and the group $T \times \mathbb{C}^{*}$ acts naturally on $\mathfrak{Q}_{\underline{d}}$. The set of fixed points of $T \times \mathbb{C}^{*}$ on $\mathfrak{Q}_{\underline{d}}$ is finite; its description is absolutely similar to [17, 2.2, which we presently recall.

Let $\underline{\vec{d}}$ be a collection of nonnegative integral vectors $\vec{d}_{i j}=\left(d_{i j}^{(1)}, \ldots, d_{i j}^{\left(p_{j}\right)}\right), n-1 \geq i \geq$ $j \geq 1$, such that $d_{i}=\sum_{j=1}^{i}\left|d_{i j}\right|=\sum_{j=1}^{i} \sum_{l=1}^{p_{j}} d_{i j}^{(l)}$, and for $i \geq k \geq j$ we have $\vec{d}_{k j} \geq \vec{d}_{i j}$, i.e. for any $1 \leq l \leq p_{j}$ we have $d_{k j}^{(l)} \geq d_{i j}^{(l)}$. Abusing notation we denote by $\underline{\vec{d}}$ the corresponding $T \times \mathbb{C}^{*}$-fixed point in $\mathfrak{Q}_{\underline{d}}$ :

$$
\begin{gathered}
\mathcal{W}_{1}=\mathcal{O}_{\mathbf{C}}\left(-d_{11}^{(1)} \cdot 0\right) w_{1} \oplus \ldots \oplus \mathcal{O}_{\mathbf{C}}\left(-d_{11}^{\left(p_{1}\right)} \cdot 0\right) w_{p_{1}}, \\
\mathcal{W}_{2}=\mathcal{O}_{\mathbf{C}}\left(-d_{21}^{(1)} \cdot 0\right) w_{1} \oplus \ldots \oplus \mathcal{O}_{\mathbf{C}}\left(-d_{21}^{\left(p_{1}\right)} \cdot 0\right) w_{p_{1}} \oplus \mathcal{O}_{\mathbf{C}}\left(-d_{22}^{(1)} \cdot 0\right) w_{p_{1}+1} \oplus \ldots \\
\ldots \oplus \mathcal{O}_{\mathbf{C}}\left(-d_{22}^{\left(p_{2}\right)} \cdot 0\right) w_{p_{1}+p_{2}}, \\
\ldots \ldots, \\
\ldots \mathcal{W}_{n-1}=\mathcal{O}_{\mathbf{C}}\left(-d_{n-1,1}^{(1)} \cdot 0\right) w_{1} \oplus \ldots \oplus \mathcal{O}_{\mathbf{C}}\left(-d_{n-1,1}^{\left(p_{1}\right)} \cdot 0\right) w_{p_{1}} \oplus \ldots \\
\ldots \oplus \mathcal{O}_{\mathbf{C}}\left(-d_{n-1, n-1}^{(1)} \cdot 0\right) w_{p_{1}+\ldots+p_{n-2}+1} \oplus \ldots \oplus \mathcal{O}_{\mathbf{C}}\left(-d_{n-1, n-1}^{\left(p_{n-1}\right)} \cdot 0\right) w_{p_{1}+\ldots+p_{n-1}} .
\end{gathered}
$$

4.5. Correspondences. For $i \in\{1, \ldots, n-1\}$, and $\underline{d}=\left(d_{1}, \ldots, d_{n-1}\right)$, we set $\underline{d}+i:=$ $\left(d_{1}, \ldots, d_{i}+1, \ldots, d_{n-1}\right)$. We have a correspondence $\mathcal{E}_{\underline{d}, i} \subset \mathcal{Q}_{\underline{d}} \times Q_{\underline{d}+i}$ formed by the pairs $\left(\mathcal{W}_{\bullet}, \mathcal{W}_{\bullet}^{\prime}\right)$ such that for $j \neq i$ we have $\mathcal{W}_{j}=\mathcal{W}_{j}^{\prime}$, and $\mathcal{W}_{i}^{\prime} \subset \mathcal{W}_{i}$, cf. [17], 2.3. In other words, $\mathcal{E}_{\underline{d}, i}$ is the moduli space of flags of locally free sheaves

$$
0 \subset \mathcal{W}_{1} \subset \ldots \mathcal{W}_{i-1} \subset \mathcal{W}_{i}^{\prime} \subset \mathcal{W}_{i} \subset \mathcal{W}_{i+1} \ldots \subset \mathcal{W}_{n-1} \subset \mathcal{W}
$$

such that $\operatorname{rank}\left(\mathcal{W}_{k}\right)=p_{1}+\ldots+p_{k}$, and $\operatorname{deg}\left(\mathcal{W}_{k}\right)=-d_{k}$, while $\operatorname{rank}\left(\mathcal{W}_{i}^{\prime}\right)=p_{1}+\ldots+p_{i}$, and $\operatorname{deg}\left(\mathcal{W}_{i}^{\prime}\right)=-d_{i}-1$.

According to 21], 2.10, $\mathcal{E}_{\underline{d}, i}$ is a smooth projective algebraic variety of dimension $\operatorname{dim}(G / P)+\sum_{i=1}^{n-1} d_{i}\left(p_{i}+p_{i+1}\right)+p_{i}$

We denote by $\mathbf{p}$ (resp. q) the natural projection $\mathcal{E}_{\underline{d}, i} \rightarrow \mathcal{Q}_{\underline{d}}$ (resp. $\mathcal{E}_{\underline{d}, i} \rightarrow Q_{\underline{d}+i}$ ). We also have a map $\mathbf{r}: \mathcal{E}_{\underline{d}, i} \rightarrow \mathbf{C}$,

$$
\left(0 \subset \mathcal{W}_{1} \subset \ldots \mathcal{W}_{i-1} \subset \mathcal{W}_{i}^{\prime} \subset \mathcal{W}_{i} \subset \mathcal{W}_{i+1} \ldots \subset \mathcal{W}_{n-1} \subset \mathcal{W}\right) \mapsto \operatorname{supp}\left(\mathcal{W}_{i} / \mathcal{W}_{i}^{\prime}\right)
$$

The correspondence $\mathcal{E}_{\underline{d}, i}$ comes equipped with a natural line bundle $\mathcal{L}_{i}$ whose fiber at a point

$$
\left(0 \subset \mathcal{W}_{1} \subset \ldots \mathcal{W}_{i-1} \subset \mathcal{W}_{i}^{\prime} \subset \mathcal{W}_{i} \subset \mathcal{W}_{i+1} \ldots \subset \mathcal{W}_{n-1} \subset \mathcal{W}\right)
$$

equals $\Gamma\left(\mathbf{C}, \mathcal{W}_{i} / \mathcal{W}_{i}^{\prime}\right)$. Let $q$ stand for the character of $T \times \mathbb{C}^{*}:(\underline{t}, v) \mapsto v$. We define the line bundle $\mathcal{L}_{i}^{\prime}:=q^{1-i} \mathcal{L}_{i}$ on the correspondence $\mathcal{E}_{\underline{d}, i}$, that is $\mathcal{L}_{i}^{\prime}$ and $\mathcal{L}_{i}$ are isomorphic as line bundles but the equivariant structure of $\mathcal{L}_{i}^{\prime}$ is obtained from the equivariant structure of $\mathcal{L}_{i}$ by the twist by the character $q^{1-i}$.

Finally, we have a transposed correspondence ${ }^{\top} \mathcal{E}_{\underline{d}, i} \subset \mathcal{Q}_{\underline{d}+i} \times \mathcal{Q}_{\underline{d}}$. 
4.6. Restricting to $\mathfrak{Q}_{\underline{d}} \subset \mathbb{Q}_{\underline{d}}$ we obtain the correspondence $\mathfrak{E}_{\underline{d}, i} \subset \mathfrak{Q}_{\underline{d}} \times \mathfrak{Q}_{\underline{d}+i}$ together with line bundle $\mathfrak{L}_{i}$ and the natural maps $\mathbf{p}: \mathfrak{E}_{d, i} \rightarrow \mathfrak{Q}_{\underline{d}}, \mathbf{q}: \mathfrak{E}_{\underline{d}, i} \rightarrow \mathfrak{Q}_{\underline{d}+i}, \mathbf{r}: \mathfrak{E}_{\underline{d}, i} \rightarrow \mathbf{C}-\infty$. We also have a transposed correspondence ${ }^{\top} \mathfrak{E}_{\underline{d}, i} \subset \mathfrak{Q}_{\underline{d}+i} \times \mathfrak{Q}_{\underline{d}}$. It is a smooth quasiprojective variety of dimension $\sum_{i=1}^{n-1} d_{i}\left(p_{i}+p_{i+1}\right)+p_{i}$.

4.7. We denote by ' $\mathrm{IH}_{G, P, T}$ the direct sum of equivariant (complexified) cohomology: ${ }^{\prime} \mathrm{IH}_{G, P, T}=\oplus_{\underline{d}} H_{T \times \mathbb{C}^{*}}^{\bullet}\left(\mathfrak{Q}_{\underline{d}}\right)$. It is a module over $H_{T \times \mathbb{C}^{*}}^{\bullet}(p t)=\mathbb{C}[\mathfrak{t} \oplus \mathbb{C}]=\mathbb{C}\left[x_{1}, \ldots, x_{N}, \hbar\right]$. Here $\mathfrak{t} \oplus \mathbb{C}$ is the Lie algebra of $T \times \mathbb{C}^{*}$. We define $\hbar$ as the positive generator of $H_{\mathbb{C}^{*}}^{2}(p t, \mathbb{Z})$. Similarly, we define $x_{i} \in H_{T}^{2}(p t, \mathbb{Z})$ in terms of the corresponding one-parametric subgroup. We define $\mathrm{IH}_{G, P, T}={ }^{\prime} \mathrm{IH}_{G, P, T} \otimes_{H_{T \times \mathbb{C}^{*}}^{\bullet}(p t)} \operatorname{Frac}\left(H_{T \times \mathbb{C}^{*}}^{\bullet}(p t)\right)$.

We have an evident grading

$$
\operatorname{IH}_{G, P, T}=\bigoplus_{\underline{d}} \operatorname{IH}_{G, P, T}^{\frac{d}{}}, \quad \text { where } \quad \operatorname{IH}_{G, P, T} \underline{d}_{T \times \mathbb{C}^{*}}\left(\mathfrak{Q}_{\underline{d}}\right) \otimes_{H_{T \times \mathbb{C}^{*}}(p t)} \operatorname{Frac}\left(H_{T \times \mathbb{C}^{*}}^{\bullet}(p t)\right) .
$$

According to the Thomason localization theorem, restriction to the $T \times \mathbb{C}^{*}$-fixed point set induces an isomorphism

$$
H_{T \times \mathbb{C}^{*}}^{\bullet}\left(\mathfrak{Q}_{\underline{d}}\right) \otimes_{H_{T \times \mathbb{C}^{*}}^{\bullet}(p t)} \operatorname{Frac}\left(H_{T \times \mathbb{C}^{*}}^{\bullet}(p t)\right) \rightarrow H_{T \times \mathbb{C}^{*}}^{\bullet}\left(\mathfrak{Q}_{\underline{d}}^{T \times \mathbb{C}^{*}}\right) \otimes_{H_{T \times \mathbb{C}^{*}}(p t)} \operatorname{Frac}\left(H_{T \times \mathbb{C}^{*}}^{\bullet}(p t)\right)
$$

The fundamental cycles $[\underline{\vec{d}}]$ of the $T \times \mathbb{C}^{*}$-fixed points $\underline{\vec{d}}$ (see Section 4.4) form a basis in $\left.\oplus_{\underline{d}} H_{T \times \mathbb{C}^{*}}^{\bullet}\left(\mathfrak{Q}_{\underline{d}}^{T \times \mathbb{C}^{*}}\right) \otimes_{H_{T \times \mathbb{C}^{*}}^{\bullet}} \operatorname{ctt}\right) \operatorname{Frac}\left(H_{T \times \mathbb{C}^{*}}^{\bullet}(p t)\right)$. The embedding of a point $\underline{\vec{d}}$ into $\mathfrak{Q}_{\underline{d}}$ is a proper morphism, so the direct image in the equivariant cohomology is well defined, and we will denote by $[\underline{\vec{d}}] \in \mathrm{IH}_{G, P, T}$ the direct image of the fundamental cycle of the point $\underline{\vec{d}}$. The set $\{[\underline{\vec{d}}]\}$ forms a basis of $\mathrm{IH}_{G, P, T}$.

4.8. For any $0 \leq i \leq n$ we will denote by $\underline{\mathcal{W}}_{i}$ the tautological $\left(p_{1}+\ldots+p_{i}\right)$-dimensional vector bundle on $\mathfrak{Q}_{\underline{d}} \times \mathbf{C}$. By the Künneth formula we have $H_{T \times \mathbb{C}^{*}}^{\bullet}\left(\mathfrak{Q}_{\underline{d}} \times \mathbf{C}\right)=H_{T \times \mathbb{C}^{*}}^{\bullet}\left(\mathfrak{Q}_{\underline{d}}\right) \otimes$ $1 \oplus H_{T \times \mathbb{C}^{*}}^{\bullet}\left(\mathfrak{Q}_{\underline{d}}\right) \otimes \tau$ where $\tau \in H_{\mathbb{C}^{*}}^{2}(\mathbf{C})$ is the first Chern class of $\mathcal{O}(1)$. Under this decomposition, for the Chern class $c_{j}\left(\underline{\mathcal{W}}_{i}\right)$ we have $c_{j}\left(\underline{\mathcal{W}}_{i}\right)=: c_{j}^{(j)}\left(\underline{\mathcal{W}}_{i}\right) \otimes 1+c_{j}^{(j-1)}\left(\underline{\mathcal{W}}_{i}\right) \otimes \tau$ where $c_{j}^{(j)}\left(\underline{\mathcal{W}}_{i}\right) \in H_{T \times \mathbb{C}^{*}}^{2 j}\left(\mathfrak{Q}_{\underline{d}}\right)$, and $c_{j}^{(j-1)}\left(\underline{\mathcal{W}}_{i}\right) \in H_{T \times \mathbb{C}^{*}}^{2 j-2}\left(\mathfrak{Q}_{\underline{d}}\right)$.

For $0 \leq m \leq n$ we introduce the generating series $\mathrm{A}_{m}(u)$ with coefficients in the equivariant cohomology ring of $\mathfrak{Q}_{\underline{d}}$ as follows:

$$
\mathrm{A}_{m}(u):=u^{p_{1}+\ldots+p_{m}}+\sum_{r=1}^{p_{1}+\ldots+p_{m}}(-\hbar)^{-r}\left(c_{r}^{(r)}\left(\underline{\mathcal{W}}_{m}\right)-\hbar c_{r}^{(r-1)}\left(\underline{\mathcal{W}}_{m}\right)\right) u^{p_{1}+\ldots+p_{m}-r}
$$

In particular, $\mathrm{A}_{0}(u):=1$.

We also define the operators

$$
\begin{gathered}
\mathrm{e}_{k}^{\left(r+1+p_{k+1}-p_{k}\right)}:=\mathbf{p}_{*}\left(c_{1}\left(\mathcal{L}_{k}^{\prime}\right)^{r} \cdot \mathbf{q}^{*}\right): \mathrm{IH}_{G, P, T}^{\frac{d}{}} \rightarrow \mathrm{IH}_{G, P, T}^{\frac{d-k}{,}}, r \geq 0 \\
\mathrm{f}_{k}^{(r+1)}:=-\mathbf{q}_{*}\left(c_{1}\left(\mathcal{L}_{k}^{\prime}\right)^{r} \cdot \mathbf{p}^{*}\right): \operatorname{IH}_{G, P, T}^{\frac{d}{d}} \rightarrow \operatorname{IH}_{G, P, T}^{\frac{d}{+}+k}, r \geq 0
\end{gathered}
$$


We consider the following generating series of operators on $\mathrm{IH}_{G, P, T}$ :

$\mathrm{d}_{k}(u)=1+\sum_{s=1}^{\infty} \mathrm{d}_{k}^{(s)} \hbar^{-s+1} u^{-s}:=\mathrm{a}_{k}(u+k-1) \mathrm{a}_{k-1}(u+k-1)^{-1}: \operatorname{IH}_{G, P, T} \rightarrow \operatorname{IH}_{G, P, T}\left[\left[u^{-1}\right]\right]$,

where $1 \leq k \leq n$ and

$$
\begin{gathered}
\mathrm{a}_{k}(u):=u^{-p_{1}}(u-1)^{-p_{2}} \ldots(u-k+1)^{-p_{k}} \mathrm{~A}_{k}(u) ; \\
\mathrm{e}_{k}(u)=\sum_{s=1+p_{k+1}-p_{k}}^{\infty} \mathrm{e}_{k}^{(s)} \hbar^{-s+1} u^{-s}: \mathrm{IH}_{G, P, T}^{\frac{d}{2}} \rightarrow \mathrm{IH}_{G, P, T}^{\frac{d-k}{2}}\left[\left[u^{-1}\right]\right], 1 \leq k \leq n-1 ; \\
\mathrm{f}_{k}(u)=\sum_{s=1}^{\infty} \mathrm{f}_{k}^{(s)} \hbar^{-s+1} u^{-s}: \operatorname{IH}_{G, P, T}^{\frac{d}{d}} \rightarrow \mathrm{IH}_{G, P, T}^{d+k}\left[\left[u^{-1}\right]\right], 1 \leq k \leq n-1 .
\end{gathered}
$$

We also introduce the auxiliary series $\mathrm{B}_{k}(u), \mathrm{C}_{k}(u)$ by the formulas (3.13), (3.14).

The following Theorem is a straightforward generalization of Theorem 2.9 and the proof of Theorem 2.12 of [17], which are in turn its particular case for $p_{1}=\ldots=p_{n}=1$.

Theorem 4.9. The matrix coefficients of the operators $\mathrm{e}_{i}^{(s)}, \mathrm{f}_{i}^{(s)}$ in the fixed point base $\{[\underline{\vec{d}}]\}$ of $\mathrm{IH}_{G, P, T}$ are as follows:

$$
\mathrm{e}_{i\left[\vec{d}, \underline{d}^{\prime}\right]}^{(s)}=\hbar^{-1}\left(\mathrm{p}_{i j}^{(a)}-i \hbar\right)^{s-1-p_{i+1}+p_{i}} \prod_{\substack{k \leq i, b \leq p_{k} \\(k, b) \neq(j, a)}}\left(\mathrm{p}_{i j}^{(a)}-\mathrm{p}_{i k}^{(b)}\right)^{-1} \prod_{k \leq i+1} \prod_{b \leq p_{k}}\left(\mathrm{p}_{i j}^{(a)}-\mathrm{p}_{i+1, k}^{(b)}\right),
$$

if $d_{i j}^{(a) \prime}=d_{i j}^{(a)}-1$ for certain $j \leq i$;

$$
\mathrm{f}_{i\left[\vec{a}, \underline{d}^{\prime}\right]}^{(s)}=-\hbar^{-1}\left(\mathrm{p}_{i j}^{(a)}+(1-i) \hbar\right)^{s-1} \prod_{\substack{k \leq i, b \leq p_{k} \\(k, b) \neq(j, a)}}\left(\mathrm{p}_{i j}^{(a)}-\mathrm{p}_{i k}^{(b)}\right)^{-1} \prod_{k \leq i-1} \prod_{b \leq p_{k}}\left(\mathrm{p}_{i j}^{(a)}-\mathrm{p}_{i-1, k}^{(b)}\right),
$$

if $d_{i j}^{(a) \prime}=d_{i j}^{(a)}+1$ for certain $j \leq i$. All the other matrix coefficients of $\mathrm{e}_{i}^{(s)}, \mathrm{f}_{i}^{(s)}$ vanish. Furthermore, the eigenvalue of $\mathrm{A}_{i}(u)$ on $[\underline{\vec{d}}]$ equals

$$
\prod_{j \leq i} \prod_{a \leq p_{j}}\left(u-\hbar^{-1} \mathbf{p}_{i j}^{(a)}\right)
$$

Proposition 4.10. The isomorphism $\Psi: \operatorname{IH}_{G, P, T} \stackrel{\sim}{\longrightarrow} V,[\underline{\vec{d}}] \mapsto(-1)^{|\underline{d}|} \hbar^{\sum_{i=1}^{n-1} d_{i} p_{i}} \xi_{\vec{d}}$ intertwines the same named operators $\mathrm{d}_{i}, \mathrm{e}_{i}, \mathrm{f}_{i}$, etc. In particular, the operators $\mathrm{d}_{i}, \mathrm{e}_{i}, \mathrm{f}_{i}$ defined in (4.3), (4.1), (4.2), turn $\mathrm{IH}_{G, P, T}$ into the Gelfand-Tsetlin module over $U^{\hbar}(\mathfrak{g}, e) \otimes \mathcal{K}^{\prime}$.

Proof. A straightforward comparison of Theorem 4.9 and Proposition 3.4 . 
4.11. Now we return to the localized $L \times \mathbb{C}^{*}$-equivariant cohomology $\mathrm{IH}_{G, P}=\mathrm{IH}_{G, P, T}^{W_{L}}$. Note that the action of $W_{L}$ on $\mathrm{IH}_{G, P, T}$ is semilinear with respect to the structure of $\mathcal{K}^{\prime}$-module, and also it commutes with the action of correspondences since both the correspondences and the line bundles $\mathcal{L}_{i}$ are equipped with the action of $L$. Hence under the identification $\Psi: \mathrm{IH}_{G, P, T} \stackrel{\sim}{\longrightarrow} V$ the $W_{L^{-}}$action on $\mathrm{IH}_{G, P, T}$ goes to the $W_{L^{-a c t i o n}}$ on $V$ introduced just before Corollary 3.6. Combining Corollary 3.6 with Proposition 4.10 we arrive at the following theorem proving Conjecture 2.9(1) in the case $\mathfrak{g}=\mathfrak{g l}_{N}$.

Theorem 4.12. The isomorphism $\Psi: \mathrm{IH}_{G, P, T} \stackrel{\sim}{\longrightarrow} V$ restricted to $W_{L}$-invariants gives the same named isomorphism of $U^{\hbar}\left(\mathfrak{g l}_{N}, e\right) \otimes \mathcal{K}$-modules $\Psi: \operatorname{IH}_{G, P} \stackrel{\sim}{\longrightarrow} M\left(\mathfrak{g l}_{N}, e\right)$.

\section{Whittaker Vector And Shapovalov Form}

Proposition 5.1. If $1_{\underline{d}}$ stands for the unit cohomology class of $\mathfrak{Q}_{\underline{d}}$, then $\Psi\left(1_{\underline{d}}\right)=\mathfrak{w}_{\underline{d}}$.

Proof. For $p_{i+1}-p_{i}+1 \leq s<p_{i+1}$, we have $\mathrm{e}_{i}^{(s)} 1_{\underline{d}}=0$ for degree reasons (it would have had a negative degree). Similarly, $\hbar \mathrm{e}_{i}^{\left(p_{i+1}\right)} 1_{\underline{d}+i}$, having degree 0 , must be a constant multiple of $1_{\underline{d}}$. More precisely, we decompose the projection $\mathbf{p}: \mathfrak{E}_{\underline{d}, i} \rightarrow \mathfrak{Q}_{\underline{d}}$ into composition of the proper $\mathbf{p} \times \mathbf{r}: \mathfrak{E}_{\underline{d}, i} \rightarrow \mathfrak{Q}_{\underline{d}} \times(\mathbf{C}-\infty)$, and further projection pr : $\overline{\mathfrak{Q}}_{\underline{d}} \times(\mathbf{C}-\infty) \rightarrow \mathfrak{Q}_{\underline{d}}$ with fibers $\mathbf{C}-\infty=\overline{\mathbb{A}}^{1}$. We have $\mathbf{p}_{*}\left(c_{1}\left(\mathcal{L}_{i}\right)^{p_{i}-1} \cdot \mathbf{q}^{*} 1_{\underline{d}+i}\right)=\operatorname{pr}_{*}(\mathbf{p} \times \mathbf{r})_{*}\left(c_{1}\left(\mathcal{L}_{i}\right)^{p_{i}-1} \cdot \mathbf{q}^{*} 1_{\underline{d}+i}\right)$. Now $(\mathbf{p} \times \mathbf{r})_{*}\left(c_{1}\left(\mathcal{L}_{i}\right)^{p_{i}-1} \cdot \mathbf{q}^{*} 1_{\underline{d}+i}\right)$ is well defined in nonlocalized equivariant cohomology, and for the degree reasons must take $1_{\underline{d}+i}$ to a constant multiple $c$ of the unit class in the equivariant cohomology of $\mathfrak{Q}_{\underline{d}} \times(\mathbf{C}-\infty)$. Furthermore, $\mathrm{pr}_{*} c=\hbar^{-1} c 1_{\underline{d}}$. So it remains to calculate the constant $c$. This can be done over the open subset $U \subset \mathfrak{Q}_{\underline{d}}$ where $\underline{\mathcal{W}}_{i} / \underline{\mathcal{W}}_{i-1}$ has no torsion, and hence $\mathbf{p} \times \mathbf{r}$ is a fibration with a fiber $\mathbb{P}^{p_{i}-1}$. More precisely, the correspondence $\mathfrak{E}_{\underline{d}, i}$ over $U \times(\mathbf{C}-\infty)$ is just the projectivized vector bundle $\mathbb{P}\left(\underline{\mathcal{W}}_{i} / \underline{\mathcal{W}}_{i-1}\right)$, and $\mathcal{L}_{i}$ is nothing else than $\mathcal{O}(1)$. We conclude that $c=1$. The proposition is proved.

Proposition 5.2. For $x, u \in \mathrm{IH}_{G, P}^{\frac{d}{}}$ we have $(\Psi(x), \Psi(u))=(-1)^{|\underline{d}|} \int_{\mathfrak{Q}_{\underline{d}}}(x u)$.

Proof. Evidently, the operators $\mathrm{f}_{i}^{(s)}$ and $-\mathrm{e}_{i}^{\left(s+p_{i+1}-p_{i}\right)}$ are adjoint with respect to the pairing $\int(? \cdot ?)$.

Thus we have fully proved Conjecture 2.9 for $\mathfrak{g}=\mathfrak{g l}(N)$.

\section{Relation to the AGt conjecture}

6.1. The Uhlenbeck spaces of $\mathbb{A}^{2}$. Let $G$ be an almost simple simply connected complex algebraic group with maximal torus $T$ and let $\mathfrak{g}, \mathfrak{t}$ be the corresponding Lie algebras. For an integer $a \geq 0$ let $\operatorname{Bun}_{G}^{d}\left(\mathbb{A}^{2}\right)$ denote the moduli space of principal $G$-bundles on $\mathbb{P}^{2}$ of second Chern class $a$ with a chosen trivialization at infinity (i.e. a trivialization on the "infinite" line $\left.\mathbb{P}_{\infty}^{1}\right)$ It is shown in [8] that this space has the following properties:

a) $\operatorname{Bun}_{G}^{d}\left(\mathbb{A}^{2}\right)$ is non-empty if and only if $a \geq 0$;

b) For $a \geq 0$ the space $\operatorname{Bun}_{G}^{d}\left(\mathbb{A}^{2}\right)$ is an irreducible smooth quasi-affine variety of dimension $2 a \check{h}$ where $\breve{h}$ denotes the dual Coxeter number of $G$.

In [8] we construct an affine scheme $\mathcal{U}_{G}^{d}\left(\mathbb{A}^{2}\right)$ containing $\operatorname{Bun}_{G}^{d}\left(\mathbb{A}^{2}\right)$ as a dense open subset which we are going to call the Uhlenbeck space of bundles on $\mathbb{A}^{2}$. 
The scheme $\mathcal{U}_{G}^{d}\left(\mathbb{A}^{2}\right)$ is still irreducible but in general it is highly singular. The main property of $\mathcal{U}_{G}^{d}\left(\mathbb{A}^{2}\right)$ is that it possesses the following stratification:

$$
\operatorname{Bun}_{G}^{d}\left(\mathbb{A}^{2}\right)=\bigcup_{0 \leq b \leq a} \operatorname{Bun}_{G}^{b}\left(\mathbb{A}^{2}\right) \times \operatorname{Sym}^{a-b}\left(\mathbb{A}^{2}\right) .
$$

Here each $\operatorname{Bun}_{G}^{b}\left(\mathbb{A}^{2}\right) \times \operatorname{Sym}^{a-b}\left(\mathbb{A}^{2}\right)$ is a locally closed subset of $U_{G}^{d}\left(\mathbb{A}^{2}\right)$ and its closure is equal to the union of similar subsets corresponding to all $b^{\prime} \leq b$.

We shall denote by $\operatorname{Bun}_{G}\left(\mathbb{A}^{2}\right)$ (resp. $\left.\mathcal{U}_{G}\left(\mathbb{A}^{2}\right)\right)$ the disjoint union of all $\operatorname{Bun}_{G}^{d}\left(\mathbb{A}^{2}\right)$ (resp. of $\left.\mathcal{U}_{G}^{d}\left(\mathbb{A}^{2}\right)\right)$.

Let us note that the group $G \times \operatorname{GL}(2)$ acts naturally on $\operatorname{Bun}_{G}^{d}\left(\mathbb{A}^{2}\right)$ : here the first factor acts by changing the trivialization at $\infty$ and the second factor acts on $\mathbb{A}^{2}$. It is easy to deduce from the construction of [8] that this action extends to an action of the same group on the Uhlenbeck space $\mathcal{U}_{G}^{d}\left(\mathbb{A}^{2}\right)$.

The group $G \times \operatorname{GL}(2)$ acts naturally on $\mathcal{U}_{G}^{d}$ where $G$ acts by changing the trivialization at $\mathbb{P}_{\infty}^{1}$ and $\operatorname{GL}(2)$ acts on $\mathbb{P}^{2}$ preserving $\mathbb{P}_{\infty}^{1}$.

6.2. Instanton counting. We may now consider the equivariant integral

$$
\int_{u_{G}^{d}} 1^{d}
$$

of the unit $G \times \mathrm{GL}(2)$-equivariant cohomology class (which we denote by $1^{d}$ ) over $\mathcal{U}_{G}^{d}$; the integral takes values in the field $\mathcal{K}$ which is the field of fractions of the algebra $\mathcal{A}=$ $H_{G \times \mathrm{GL}(2)}^{*}(p t)$. Note that $\mathcal{A}$ is canonically isomorphic to the algebra of polynomial functions on the Lie algebra $\mathfrak{g} \times \mathfrak{g l}(2)$ which are invariant with respect to the adjoint action. Thus each $\int 1^{d}$ may naturally be regarded as a rational function of $a \in \mathfrak{t}$ and $\left(\varepsilon_{1}, \varepsilon_{2}\right) \in \mathbb{C}^{2}$; this $u_{G}^{d}$

function must be invariant with respect to the natural action of $W$ on $\mathfrak{t}$ and with respect to interchanging $\varepsilon_{1}$ and $\varepsilon_{2}$.

Consider now the generating function

$$
z=\sum_{d=0}^{\infty} Q^{d} \int_{\mathcal{U}_{G}^{d}} 1^{d} .
$$

It can (and should) be thought of as a function of the variables $\mathfrak{q}$ and $a, \varepsilon_{1}, \varepsilon_{2}$ as before. The function $Z\left(Q, a, \varepsilon_{1}, \varepsilon_{2}\right)$ is called the Nekrasov partition function of pure $N=2$ supersymmetric gauge theory.

6.3. The AGT conjecture. In [2] Alday, Gaiotto and Tachikawa suggested a relation between 4-dimensional supersymmetric gauge theory for $G=\mathrm{SL}(2)$ and the so called Liouville 2-dimensional conformal field theory; some generalizations to other groups were suggested in [4, 23]. Here we are going to formulate a few mathematical statements suggested by the AGT conjecture (it is not clear to us whether from the physics point of view they should be perceived as direct corollaries of it).

Consider the above Uhlenbeck space $\mathcal{U}_{G}^{d}$ and let $\mathrm{IH}_{G \times G L(2)}\left(\mathcal{U}_{G}^{d}\right)$ denote its equivariant intersection cohomology. This is a module over the algebra $\mathcal{A}_{G \times \mathrm{GL}(2)}:=H_{G \times \mathrm{GL}(2)}^{*}(p t)$; this 
algebra is just the algebra of polynomial functions on $\mathfrak{g} \times \mathfrak{g l}(2)$ which are invariant under the adjoint action. We denote by $\mathcal{K}_{G \times \mathrm{GL}(2)}$ its field of fractions and we let

$$
\mathrm{IH}_{G}^{d, \text { aff }}=\mathrm{IH}_{G \times \mathrm{GL}(2)}^{*}\left(\mathcal{U}_{G}^{d}\right) \underset{\mathcal{A}_{G \times \mathrm{GL}(2)}}{\otimes} \mathcal{K}_{G \times \mathrm{GL}(2)} .
$$

We also set $\mathrm{IH}_{G}^{\text {aff }}$ to be the direct sum of all the $\mathrm{IH}_{G}^{d \text { aff }}$. This is a vector space over $\mathcal{K}_{G \times \mathrm{GL}(2)}$ which informally we may think of as a family of vector spaces parametrized by $a \in \mathfrak{t} / W$ and $\left(\varepsilon_{1}, \varepsilon_{2}\right) \in \mathbb{C}^{2} / \mathbb{Z}_{2}$. Also, each $\mathrm{IH}_{G}^{d}$ is endowed with a perfect symmetric pairing $\langle\cdot, \cdot\rangle$, which is equal to the Poincaré pairing multiplied by $(-1)^{\breve{h} d}$.

Consider now the case $G=\mathrm{SL}(2)$. Then we can identify the Cartan subalgebra $\mathfrak{h}$ of $\mathfrak{s l}(2)$ with $\mathbb{C}$. Thus $a \in \mathfrak{h}$ can be thought of as a complex number.

Warning. It is important to note that if we think about $a$ as a weight of the Langlands dual $\mathfrak{s l}(2)$-algebra with standard generators $e, h, f$, then by definition the value of this weight on $h$ is equal to $\alpha(a)=2 a$ (where $\alpha$ denotes the simple root of $\mathfrak{s l}(2)$. This observation will be used below.

Let Vir denote the Virasoro Lie algebra; it has the standard generators $\left\{L_{n}\right\}_{n \in \mathbb{Z}}$ and $\mathbf{c}$ where $\mathbf{c}$ is central and $L_{n}$ 's satisfy the standard relations.

Given a field $K$ of characteristic 0 , for every $\Delta \in K, c \in K$ we may consider the Verma module $\mathbf{M}_{\Delta, c}$ over Vir on which $\mathbf{c}$ acts by $c$ and which is generated by a vector $m_{\Delta, c}$ such that

$$
L_{0}\left(m_{\Delta, c}\right)=\Delta m_{\Delta, c} ; \quad L_{n}\left(m_{\Delta, c}\right)=0 \text { for } n>0 .
$$

In addition, the Verma module $\mathbf{M}_{\Delta, c}$ is equal to the direct sum of its $L_{0}$-eigen-spaces $\mathbf{M}_{\Delta, c, d}$ where $n \in \mathbb{Z}_{\geq 0}$ and $L_{0}$ acts on $\mathbf{M}_{\Delta, c, d}$ by $\Delta+d$. It is easy to see that there exists unique collection of vectors $w_{\Delta, c, d} \in \mathbf{M}_{\Delta, c, d}$ (for all $d \in \mathbb{Z}_{\geq 0}$ ) such that

1) $w_{\Delta, c, 0}=m_{\Delta, c}$;

2) We have $L_{i} \cdot w_{\Delta, c, d}=0$ for $i>1$ and $L_{1} \cdot w_{\Delta, c, d}=w_{\Delta, c, d-1}$.

We let $w_{\Delta, c}$ denote the sum of all the $w_{\Delta, c, d}$; this is an element of the completed Verma module $\hat{\mathbf{M}}_{\Delta, c}=\prod_{d \geq 0} \mathbf{M}_{\Delta, c, d}$.

In addition the module $\mathbf{M}_{\Delta, c}$ possesses unique symmetric bilinear form $\langle\cdot, \cdot\rangle$ such that $\left\langle m_{\Delta, c}, m_{\Delta, c}\right\rangle=1$ and $L_{n}$ is adjoint to $L_{-n}$.

Then the AGT conjecture implies the following (the statement below is often referred to as "non-conformal limit" of the AGT conjecture, cf. [24]):

Conjecture 6.4. Let

$$
\Delta=-\frac{a^{2}}{\varepsilon_{1} \varepsilon_{2}}+\frac{\left(\varepsilon_{1}+\varepsilon_{2}\right)^{2}}{4 \varepsilon_{1} \varepsilon_{2}} ; \quad c=1+\frac{6\left(\varepsilon_{1}+\varepsilon_{2}\right)^{2}}{\varepsilon_{1} \varepsilon_{2}} .
$$

Then

In other words,

$$
\int_{\mathcal{u}_{G}^{d}} 1=\left\langle w_{\Delta, c, d}, w_{\Delta, c, d}\right\rangle .
$$

$$
Z\left(a, \varepsilon_{1}, \varepsilon_{2},(-1)^{\check{h}} Q\right)=Q^{-\Delta}\left\langle w_{\Delta, c}, Q^{L_{0}} w_{\Delta, c}\right\rangle .
$$

In fact, it is quite natural to expect that the following stronger result holds: 
Conjecture 6.5. (1) There exists an action of the Virasoro algebra Vir on $\mathrm{IH}_{G}$ such that with this action $\mathrm{IH}_{G}^{\text {aff }}$ becomes isomorphic to $\mathbf{M}_{\Delta, c}$ where

(2) The intersection pairing is Vir-invariant, i.e. the adjoint operator to $L_{n}$ is $L_{-n}$.

(3) $L_{n} \cdot 1^{d}=0$ for any $n>1$ and $d \geq 0$.

(4) $L_{1} \cdot 1^{d}=1^{d-1}$ for any $d>0$.

One can generalize Conjecture 6.4 and Conjecture 6.5 to arbitrary $G$. We are not going to give details here, but let us stress one thing: when $G$ is simply laced the Virasoro algebra Vir has to be replaced by the $W$-algebra corresponding to the affine Lie algebra $\mathfrak{g}_{\text {aff }}$; in fact for general $G$ (not necessarily simply laced) we believe that the $W$-algebra associated with the Langlands dual affine Lie algebra $\mathfrak{g}_{\text {aff }}^{\vee}$ should appear (cf. the next subsection for some motivation). In other words, we expect that for general $G$ the space $\mathrm{IH}_{G}$ carries a natural action of the $W$-algebra $W\left(\mathfrak{g}_{\text {aff }}^{\vee}\right)$, which makes it into a Verma module over this algebra; analogs of properties 2,3,4 above are also expected 2 . It is easy to deduce from the results of [8] that the character of $\mathrm{IH}_{G}$ is equal to the character of the Verma module for the $W$-algebra.

Before we explain the connection of Conjecture 6.5 with the rest of the paper, let us recall some (known) modification of it.

6.6. The flag case. Choose a parabolic subgroup $P \subset G$ and let $\mathbf{C}$ denote the "horizontal" line in $\mathbb{P}^{2}$ (i.e. we choose a straight line in $\mathbb{P}^{2}$ different from the one at infinity and call). Let $\operatorname{Bun}_{G, P}$ denote the moduli space of the following objects:

1) A principal $G$-bundle $\mathcal{F}_{G}$ on $\mathbb{P}^{2}$;

2) A trivialization of $\mathcal{F}_{G}$ on $\mathbb{P}_{\infty}^{1}$;

3) A reduction of $\mathcal{F}_{G}$ to $P$ on $\mathbf{C}$ compatible with the trivialization of $\mathcal{F}_{G}$ on $\mathbf{C}$.

Let us describe the connected components of $\operatorname{Bun}_{G, P}$. We are going to use the notations of Section 1.1. Let also $\Lambda_{G, P}^{\mathrm{aff}}=\Lambda_{G, P} \times \mathbb{Z}$ be the lattice of characters of $Z(\check{L}) \times \mathbb{C}^{*}$. Note that $\Lambda_{G, G}^{\mathrm{aff}}=\mathbb{Z}$.

The lattice $\Lambda_{G, P}^{\text {aff }}$ contains canonical semi-group $\Lambda_{G, P}^{\text {aff, }+}$ of positive elements (cf. [8]). It is not difficult to see that the connected components of $\operatorname{Bun}_{G, P}$ are parameterized by the elements of $\Lambda_{G, P}^{\mathrm{aff},+}$ :

$$
\operatorname{Bun}_{G, P}=\bigcup_{\theta_{\mathrm{aff}} \in \Lambda_{G, P}^{\mathrm{aff},+}} \operatorname{Bun}_{G, P}^{\theta_{\mathrm{aff}}} .
$$

Typically, for $\theta_{\text {aff }} \in \Lambda_{G, P}^{\text {aff }}$ we shall write $\theta_{\text {aff }}=(\theta, d)$ where $\theta \in \Lambda_{G, P}$ and $d \in \mathbb{Z}$.

Each $\operatorname{Bun}_{G, P}^{\theta_{\text {aff }}}$ is naturally acted upon by $P \times\left(\mathbb{C}^{*}\right)^{2}$; embedding $L$ into $P$ we get an action of $L \times\left(\mathbb{C}^{*}\right)^{2}$ on $\operatorname{Bun}_{G, P}^{\theta_{\text {aff }}}$. In [8] we define for each $\theta_{\text {aff }} \in \Lambda_{G, P}^{\text {aff, }+}$ certain Uhlenbeck scheme $\mathcal{U}_{G, P}^{\theta_{\text {aff }}}$ which contains $\operatorname{Bun}_{G, P}^{\theta_{\text {aff }}}$ as a dense open subset. The scheme $\mathcal{U}_{G, P}^{\theta_{\text {aff }}}$ still admits an action of $L \times\left(\mathbb{C}^{*}\right)^{2}$.

\footnotetext{
${ }^{2}$ A modification of this conjecture exists also for $\mathfrak{g}=\mathfrak{g l}(N)$; this conjecture will be proved in 25 .
} 
Following [5] define

$$
z_{G, P}^{\mathrm{aff}}=\sum_{\theta \in \Lambda_{G, P}^{\mathrm{aff}}} \mathfrak{q}_{\mathrm{aff}}^{\theta_{\mathrm{aff}}} \int_{u_{G, P}^{\theta_{\text {aff }}}} 1_{G, P}^{\theta_{\text {aff }}} .
$$

Remark. In addition to [5] and [7] various examples of functions $z_{G, P}^{\text {aff }}$ were studied recently in the physical literature (cf. for example [1, [3]) as (the instanton part of) the Nekrasov partition function in the presence of surface operators.

One should think of $Z_{G, P}^{\text {aff }}$ as a formal power series in $\mathfrak{q}_{\text {aff }} \in Z(\check{L}) \times \mathbb{C}^{*}$ with values in the space of ad-invariant rational functions on $\mathfrak{l} \times \mathbb{C}^{2}$. Typically, we shall write $\mathfrak{q}_{\text {aff }}=(\mathfrak{q}, Q)$ where $\mathfrak{q} \in Z(\check{L})$ and $Q \in \mathbb{C}^{*}$. Also we shall denote an element of $\mathfrak{l} \times \mathbb{C}^{2}$ by $\left(a, \varepsilon_{1}, \varepsilon_{2}\right)$ or (sometimes it will be more convenient) by $(a, \hbar, \varepsilon)$ (note that for general $P$ (unlike in the case $P=G$ ) the function $Z_{G, P}^{\text {aff }}$ is not symmetric with respect to switching $\varepsilon_{1}$ and $\varepsilon_{2}$ ).

As before, let us now denote by $\mathrm{IH}_{G, P}^{\theta_{\text {aff }}}$ the localized $L \times\left(\mathbb{C}^{*}\right)^{2}$-equivariant intersection cohomology of $U_{G, P}^{\theta_{\text {aff }}}$; we also set $\mathrm{IH}_{G, P}^{\text {aff }}$ to be the direct sum of all the $\mathrm{IH}_{G, P}^{\theta_{\text {aff }}}$; note that $\mathrm{IH}_{G, G}^{\mathrm{aff}}=\mathrm{IH}_{G}^{\mathrm{aff}}$. Then $\mathrm{IH}_{G, P}^{\mathrm{aff}}$ is $\Lambda_{G, P}^{\mathrm{aff},+}$-graded $\mathcal{K}_{L \times\left(\mathbb{C}^{*}\right)^{2}}$ vector space. We can endow with a non-degenerate pairing $\langle\cdot, \cdot\rangle$

The following result is proved in [5]:

Theorem 6.7. Let $P=B$ be a Borel subgroup. Then $\mathrm{IH}_{G, B}^{\mathrm{aff}}$ possesses a natural action of the Lie algebra $\check{\mathfrak{g}}_{\text {aff }}$ such that

(1) As a $\check{\mathfrak{g}}_{\text {aff }}$-module $\mathrm{IH}_{G, B}^{\text {aff }}$ is isomorphic to $M\left(\lambda_{\text {aff }}\right)$, where $\lambda_{\text {aff }}=-\frac{\left(a, \varepsilon_{1}\right)}{\varepsilon_{2}}-\rho_{\text {aff }}$.

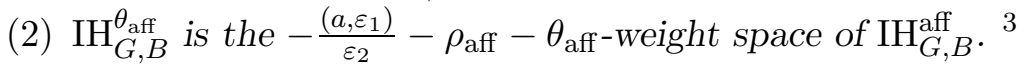

(3) The isomorphism of (1) takes the pairing $\langle\cdot, \cdot\rangle$ on $\operatorname{IH}_{G, B}^{\theta_{\text {aff }}}$ to $(-1)^{\left|\theta_{\text {aff }}\right|}$ times the

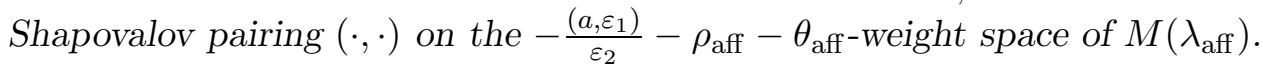

6.8. Interpretation via maps and the "finite-dimensional" analog. Choose now another smooth projective curve $\mathbf{X}$ of genus 0 and with two marked points $0 \mathbf{X}, \infty \mathbf{X}$. Choose also a coordinate $x$ on $\mathbf{X}$ such that $x\left(0_{\mathbf{X}}\right)=0$ and $x\left(\infty_{\mathbf{X}}\right)=0$. Let us denote by $\mathcal{G}_{G, P, \mathbf{X}}$ the scheme classifying triples $\left(\mathcal{F}_{G}, \beta, \gamma\right)$, where

1) $\mathcal{F}_{G}$ is a principal $G$-bundle on $\mathbf{X}$;

2) $\beta$ is a trivialization of $\mathcal{F}_{G}$ on the formal neighborhood of $\infty_{\mathbf{X}}$;

3) $\gamma$ is a reduction to $P$ of the fiber of $\mathcal{F}_{G}$ at $0 \mathbf{X}$.

We shall usually omit $\mathbf{X}$ from the notations. We shall also write $\mathcal{G}_{G}^{\text {aff }}$ for $\mathcal{G}_{G, G}^{\text {aff }}$.

Let $e_{G, P}^{\text {aff }} \in \mathcal{G}_{G, P}^{\text {aff }}$ denote the point corresponding to the trivial $\mathcal{F}_{G}$ with the natural $\beta$ and $\gamma$. It is explained in [8] that the variety $\operatorname{Bun}_{G, P}$ is canonically isomorphic to the scheme classifying based maps from $\left(\mathbb{P}^{1}, \infty\right)$ to $\left(\mathcal{G}_{G, P}^{\text {aff }}, e_{G, P}^{\text {aff }}\right)$ (i.e. maps from $\mathbb{P}^{1}$ to $\mathcal{G}_{G, P}$ sending $\infty$ to $\left.e_{G, P}^{\text {aff }}\right)$.

The scheme $\mathcal{G}_{G, P}^{\text {aff }}$ may (and should) be thought of as a partial flag variety for $\mathfrak{g}_{\text {aff }}$. Thus the scheme $\operatorname{Bun}_{G, P}$ should be thought of as an affine analog of $\mathcal{M}_{G, P}$. Also the flag Uhlenbeck

\footnotetext{
${ }^{3}$ Here by $\left(a, \varepsilon_{1}\right)$ we mean the weight of $\mathfrak{g}_{\text {aff }}^{\vee}$ whose "finite" component is $a$ and whose central charge is $\varepsilon_{1}$. Also $\rho_{\text {aff }}$ is a weight of $\mathfrak{g}_{\text {aff }}^{\vee}$ which takes value 1 on every simple coroot.
} 
scheme $\mathcal{U}_{G, P}$ should be thought of as an affine analog of the scheme $Q M_{G, P}$ (this analogy is explained in more detail in [8]).

Thus Theorem 6.7 can be considered as an affine version of Theorem 1.4 and Conjecture 6.5 (together with its generalization to arbitrary $\mathfrak{g}$ mentioned above) is an affine version of Conjecture 2.9 (in fact, there should be a more general version of this conjecture, dealing not only with $\mathrm{IH}_{G}^{\text {aff }}$ but with arbitrary $\mathrm{IH}_{G, P}^{\text {aff }}$ ). To conclude the paper we are going to explain how to use this analogy with Conjecture 2.9 in order to derive the formulas (6.2) from Theorem 6.7. For $\chi, k \in \mathbb{C}$ let $M(\chi, k)$ denote the Verma module over $\mathfrak{s l}(2)_{\text {aff }}$ with central charge $k$ and highest weight $\chi$ (i.e. the standard generator $h$ of $\mathfrak{s l}(2)$ acts on the highest weight vector as multiplication by $\chi)$. According to [14] the algebra Vir is obtained by certain BRST reeduction from $U\left(\mathfrak{s l}(2)_{\text {aff }}\right)$; the corresponding BRST reduction of $M(\chi, k)$ is equal to $\mathbf{M}_{\Delta, c}$ where

$$
\Delta=\frac{(\chi+1)^{2}-(k+1)^{2}}{4(k+2)} ; \quad c=1-\frac{6(k+1)^{2}}{k+2} .
$$

According to Theorem 6.7 we should take

$$
\chi=-\frac{2 a}{\varepsilon_{2}}-1 ; \text {; } \quad k=-\frac{\varepsilon_{1}}{\varepsilon_{2}}-2 .
$$

Thus $k+2=-\varepsilon_{1} / \varepsilon_{2}$ and

Hence

$$
\frac{(k+1)^{2}}{k+2}=-\frac{\left(\varepsilon_{1}+\varepsilon_{2}\right)^{2}}{\varepsilon_{1} \varepsilon_{2}} .
$$

and

$$
c=1+\frac{6\left(\varepsilon_{1}+\varepsilon_{2}\right)^{2}}{\varepsilon_{1} \varepsilon_{2}}
$$

$$
\Delta=\frac{(\chi+1)^{2}-(k+1)^{2}}{4(k+2)}=-\frac{a^{2}}{\varepsilon_{1} \varepsilon_{2}}+\frac{\left(\varepsilon_{1}+\varepsilon_{2}\right)^{2}}{4 \varepsilon_{1} \varepsilon_{2}},
$$

which coincides with (6.2).

\section{REFERENCES}

[1] L. F. Alday, D. Gaiotto, S. Gukov, Y. Tachikawa, H. Verlinde, Loop and surface operators in $N=2$ gauge theory and Liouville modular geometry, arXiv:0909.0945

[2] L. F. Alday, D. Gaiotto and Y. Tachikawa, Liouville correlation functions from four-dimensional gauge theories, Lett. Math. Phys. 91 (2010), no. 2, 167-197.

[3] L. F. Alday, Y. Tachikawa, Affine SL(2) conformal blocks from 4d gauge theories, arXiv:1005.4469

[4] H. Awata, Y. Yamada, Five-dimensional AGT Relation and the deformed $\beta$-ensemble, preprint arXiv:1004.5122.

[5] A. Braverman, Instanton counting via affine Lie algebras. I. Equivariant J-functions of (affine) flag manifolds and Whittaker vectors, Algebraic structures and moduli spaces, CRM Proc. Lecture Notes 38 Amer. Math. Soc., Providence, RI (2004), 113-132.

[6] A. Braverman, Spaces of quasi-maps and their applications, International Congress of Mathematicians. Vol. II, Eur. Math. Soc., Zürich, (2006), 1145-1170.

[7] A. Braverman and P. Etingof, Instanton counting via affine Lie algebras II: from Whittaker vectors to the Seiberg-Witten prepotential, Studies in Lie theory, 61-78, Progr. Math., 243, Birkhäuser Boston, Boston, MA, 2006.

\footnotetext{
${ }^{4}$ The appearance of the factor 2 is explained in the Warning in Section 6.3
} 
[8] A. Braverman, M. Finkelberg and D. Gaitsgory, Uhlenbeck spaces via affine Lie algebras, in: The unity of mathematics (volume dedicated to I. M. Gelfand's 90th birthday), Progr. Math., 244, Birkhäuser Boston (2006), 17-135.

[9] A. Braverman, M. Finkelberg, D. Gaitsgory, I. Mirković, Intersection cohomology of Drinfeld's compactifications. Selecta Math. (N.S.) 8 (2002), no. 3, 381-418.

[10] J. Brundan, S. M. Goodwin, Good grading polytopes, Proc. London Math. Soc. 94 (2007), 155-180.

[11] J. Brundan, S. Goodwin, A. Kleshchev, Highest weight theory for finite W-algebras, Int. Math. Res. Not. (2008) no. 15, Art. ID rnn051, 53 pp.

[12] J. Brundan, A. Kleshchev, Representations of shifted Yangians and finite W-algebras, Mem. Amer. Math. Soc. 196, no. 918 (2008), viii+107pp.

[13] P. Etingof, Whittaker functions on quantum groups and q-deformed Toda operators, in: Differential topology, infinite-dimensional Lie algebras, and applications, Amer. Math. Soc. Transl. Ser. 2, 194, Amer. Math. Soc., Providence, RI, (1999), 9-25.

[14] B. Feigin and E. Frenkel, Representations of affine Kac-Moody algebras, bosonization and resolutions, Lett. Math. Phys. 19 (1990), 307-317.

[15] M. Finkelberg and I. Mirković, Semi-infinite flags. I. Case of global curve $\mathbb{P}^{1}$, in: Differential topology, infinite-dimensional Lie algebras, and applications, Amer. Math. Soc. Transl. Ser. 2, 194, Amer. Math. Soc., Providence, RI, (1999), 81-112.

[16] Feigin, B., Finkelberg, M., Kuznetsov, A., Mirković, I., Semi-infinite flags. II. Local and global intersection cohomology of quasimaps' spaces, in: Differential topology, infinite-dimensional Lie algebras, and applications, Amer. Math. Soc. Transl. Ser. 2, 194, Amer. Math. Soc., Providence, RI, (1999), $113-148$.

[17] B. Feigin, M. Finkelberg, A. Negut, L. Rybnikov, Yangians and cohomology rings of Laumon spaces, preprint arXiv:0812.4656

[18] V. Futorny, A. Molev, S. Ovsienko, Gelfand-Tsetlin bases for representations of finite $W$-algebras and shifted Yangians, in "Lie theory and its applications in physics VII", (H. D. Doebner and V. K. Dobrev, Eds), Proceedings of the VII International Workshop, Varna, Bulgaria, June 2007. Heron Press, Sofia (2008), 352-363, see also arXiv:0711.0552

[19] A. Givental, B. Kim, Quantum cohomology of flag manifolds and Toda lattices, Comm. Math. Phys. 168 (1995), no. 3, 609-641.

[20] B. Kim, Quantum cohomology of flag manifolds $G / B$ and quantum Toda lattices, Ann. of Math. (2) 149 (1999), 129-148.

[21] G. Laumon, Un Analogue Global du Cône Nilpotent, Duke Math. Journal 57 (1988), 647-671.

[22] G. Laumon, Faisceaux Automorphes Liés aux Séries d'Eisenstein, Perspect. Math. 10 (1990), $227-281$.

[23] A. Mironov, A. Morozov, On AGT relation in the case of U(3), Nucl.Phys.B 825 (2010), 1-37.

[24] A. Marshakov, A. Mironov and A. Morozov, On non-conformal limit of the AGT relations, Phys. Lett. B 682 (2009), no. 1, 125-129.

[25] D. Maulik and A. Okounkov, in preparation.

[26] M. Taki, On AGT Conjecture for Pure Super Yang-Mills and W-algebra, preprint arXiv:0912.4789.

A.B.: Department of Mathematics, Brown University, 151 Thayer St., Providence RI 02912, USA; braval@math. brown.edu

B.F.: Landau Institute and State University Higher School of Economics Department of Mathematics, 20 Myasnitskaya st, Moscow 101000 Russia;

bfeigin@gmail.com

M.F.: IMU, IITP and State University Higher School of Economics

Department of Mathematics, 20 Myasnitskaya st, Moscow 101000 Russia;

fnklberg@gmail.com

L.R.: IITP and State University Higher School of Economics

Department of Mathematics, 20 Myasnitskaya st, Moscow 101000 Russia;

leo.rybnikov@gmail.com 